\title{
Irreversible JNK1-JUN inhibition by JNK-IN-8 sensitizes pancreatic cancer to 5-FU/FOLFOX chemotherapy
}

\author{
Matthew B. Lipner, ${ }^{1,2}$ Xianlu L. Peng, ${ }^{2}$ Chong Jin,, ${ }^{2,3}$ Yi Xu, ${ }^{2}$ Yanzhe Gao, ${ }^{2,4}$ Michael P. East, ${ }^{1,2}$ \\ Naim U. Rashid, ${ }^{2,3}$ Richard A. Moffitt, ${ }^{1,2}$ Silvia G. Herrera Loeza, ${ }^{2}$ Ashley B. Morrison, ${ }^{2}$ \\ Brian T. Golitz, ${ }^{1}$ Cyrus Vaziri, ${ }^{2,4}$ Lee M. Graves, ${ }^{1,2}$ Gary L. Johnson, ${ }^{1,2}$ and Jen Jen Yeh ${ }^{1,2,5}$ \\ Department of Pharmacology, ${ }^{2}$ Lineberger Comprehensive Cancer Center, ${ }^{3}$ Department of Biostatistics, ${ }^{4}$ Department of \\ Pathology, and ${ }^{5}$ Department of Surgery, School of Medicine, University of North Carolina at Chapel Hill, Chapel Hill, \\ North Carolina, USA.
}

Over 55,000 people in the United States are diagnosed with pancreatic ductal adenocarcinoma (PDAC) yearly, and fewer than $\mathbf{2 0} \%$ of these patients survive a year beyond diagnosis. Chemotherapies are considered or used in nearly every PDAC case, but there is limited understanding of the complex signaling responses underlying resistance to these common treatments. Here, we take an unbiased approach to study protein kinase network changes following chemotherapies in patient-derived xenograft (PDX) models of PDAC to facilitate design of rational drug combinations. Proteomics profiling following chemotherapy regimens reveals that activation of JNK-JUN signaling occurs after 5-fluorouracil plus leucovorin (5-FU + LEU) and FOLFOX (5-FU + LEU plus oxaliplatin [OX]), but not after $\mathrm{OX}$ alone or gemcitabine. Cell and tumor growth assays with the irreversible inhibitor JNK-IN-8 and genetic manipulations demonstrate that JNK and JUN each contribute to chemoresistance and cancer cell survival after FOLFOX. Active JNK1 and JUN are specifically implicated in these effects, and synergy with JNK-IN-8 is linked to FOLFOX-mediated JUN activation, cell cycle dysregulation, and DNA damage response. This study highlights the potential for JNK-IN-8 as a biological tool and potential combination therapy with FOLFOX in PDAC and reinforces the need to tailor treatment to functional characteristics of individual tumors.

Conflict of interest: The authors have declared that no conflict of interest exists.

Copyright: () 2020, American Society for Clinical Investigation.

Submitted: May 3, 2019

Accepted: March 18, 2020

Published: March 26, 2020

Reference information: /CI Insight. 2020;5(8):e129905.

https://doi.org/10.1172/jci.

insight.129905.

\section{Introduction}

Pancreatic ductal adenocarcinoma (PDAC) is the third leading cause of cancer deaths in the United States, in part because over $80 \%$ of patients are diagnosed at the outset with locally advanced or metastatic disease for which surgical resection is not an option $(1,2)$. Targeted inhibitors have revolutionized clinical care for many cancers but not for PDAC, a heterogeneous and aggressive disease that has failed to meaningfully respond to any targeted therapies despite increasing understanding of its genetic and biologic drivers. Significant efforts have instead been taken to evaluate combinations of existing chemotherapies as treatment regimens, and several of these combinations have demonstrated improved patient survival over individual agents and have become preferred treatments for PDAC Despite these successes and new combinations under evaluation, functions of specific signaling networks in mediating response and resistance to these treatments remain incompletely understood. There is an urgent need for new methods and approaches for determining rational drug combinations with these widely used chemotherapies.

FOLFOX is one of the recommended chemotherapy combinations for PDAC and consists of (a) 5-fluorouracil (5-FU), a pyrimidine analog that blocks thymidine synthesis and, thereby, DNA replication; (b) leucovorin (LEU), a vitamin B9 derivative that improves efficacy of 5-FU, and (c) oxaliplatin (OX), a platinum-based chemotherapy agent that creates DNA cross links to interfere with DNA replication and repair. FOLFOX was shown to be more effective than 5-FU or OX individually, and the combination has been generally well tolerated in clinical settings (3-5). Retrospective studies have indicated that patients respond similarly to FOLFOX as they do to other recommended combinations, including regimens containing the mechanistically similar, often 
prescribed chemotherapeutic agent gemcitabine (GEM) $(6,7)$. Current guidelines recommend FOLFOX as an option for patients with GEM refractory PDAC (8).

FOLFOX itself is also a promising candidate for further drug combination, as it has been combined with the chemotherapy irinotecan to form FOLFIRINOX, the first-line treatment for both adjuvant chemotherapy and metastatic PDAC $(9,10)$. This combination's greatly increased efficacy was a breakthrough in PDAC, but the addition of the cytotoxic irinotecan leads to increased frequency and severity of side effects (9-11). Therefore, at many centers, it is currently only recommended to healthier, younger patients, which may exclude as many as $75 \%$ of metastatic PDAC patients (12).

While many drug uptake and metabolic mechanisms for resistance to 5-FU and GEM are known $(13,14)$, there is much less understanding of the signaling pathway changes, especially in the global set of protein kinases (i.e., the "kinome") that mediate cell response and survival after treatment with these widely used chemotherapy regimens in $\operatorname{PDAC}(13,15)$. Here, we describe an unbiased approach to assessing potential kinase targets for inhibition in combination with FOLFOX. We subsequently validate JNK1 and JUN as kinase mediators of FOLFOX resistance that can be therapeutically exploited with irreversible JNK inhibition by the recently developed drug JNK-IN-8 (16).

Roles for JNK in specific cancer types remain elusive and complex, as JNK regulates both tumor-suppressive responses to UV exposure and apoptosis (17-19), as well as oncogenic proliferative and invasive functions through phosphorylation of its main substrate, the transcription factor c-JUN (17, 19-22). JNK possesses similarly multifaceted roles in PDAC, with verified tumor-suppressive $(23,24)$ and tumor-promoting functions (25-27) that may be dependent upon a number of factors, including the stage of tumor initiation/development being assessed and whether JNK is being studied in the context of another treatment. JUN has been found to be overexpressed in PDAC (28), prompting testing of JNK inhibition as a therapeutic approach in PDAC $(25,29,30)$, but most of these studies have relied upon the reversible inhibitor SP600125 that has been proven to be highly nonspecific. SP600125 inhibits a wide range of kinases, including p38, CDK1, and MEK/ERK - all pathways with connections to JNK signaling that could be confounding (31-33). Moreover, lack of inhibitor specificity has hindered understanding of individual roles in PDAC biology for JNK1 and JNK2, which are known to act both in concert and direct opposition in different cancer types.

In this study, we assessed contributions of JNK1, JNK2, and JUN in the response to 5-FU and FOLFOX chemotherapies in PDAC by evaluating signaling and phenotypic consequences of pharmacological inhibition of JNK with JNK-IN-8. We determined that JNK-IN-8 is highly specific, rapidly effective, and able to durably inhibit JUN phosphorylation in PDAC cells and patient-derived tumors. JNK-IN-8 enhanced FOLFOX inhibition of PDAC tumor and cell growth, synergy that may be facilitated by FOLFOX-induced DNA damage response and cell cycle dysregulation. Genetic manipulations of PDAC cell lines demonstrated that JUN is necessary for mediating resistance to FOLFOX and sensitivity to JNKIN-8. Furthermore, JNK1 and JUN, unlike JNK2, are sufficient to induce further resistance to FOLFOX and abrogate synergy between FOLFOX and JNK-IN-8, establishing the JNK1-JUN pathway as a critical and therapeutically vulnerable mediator of chemotherapy response in PDAC.

\section{Results}

5-FU and FOLFOX chemotherapy regimens induce druggable activation of JNK and JUN. Comprehensive signaling responses and vulnerabilities following chemotherapy treatments in PDAC have not been unraveled. 5-FU is frequently used as the backbone for combinatorial therapies and is part of both first- and second-line treatments, including FOLFOX $(3-5,8-10)$. Therefore, we performed unbiased profiling of the expression of the kinome following FOLFOX and single cytotoxic agents (5-FU, OX, and GEM) in PDAC patient-derived xenograft (PDX) tumors. The functional kinome following chemotherapies was analyzed using multiplexed-kinase inhibitor bead chromatography with mass spectrometry (MIB-MS), a technique in which endogenous kinases are captured and quantified from cell or tumor lysates (Figure 1A) $(34,35)$. Over 300 unique kinases were captured across all tumors. Among upregulated kinases compared with pretreatment biopsies for 5-FU or vehicle-treated tumors for FOLFOX, there was a marked enrichment for signaling components involved in JNK signaling, including JNK1 (MAPK8), JNK2 (MAPK9), and multiple upstream kinases that activate JNK such as MAP4K4, MAP3K1, TAOK1 (MAP3K16), and TAOK2 (MAP3K17) (19, 36-39). In contrast, treatment with OX alone or GEM, a mechanistically similar chemotherapy to 5-FU, did not lead to widespread upregulation of JNK1/2 or known JNK activators. 
A
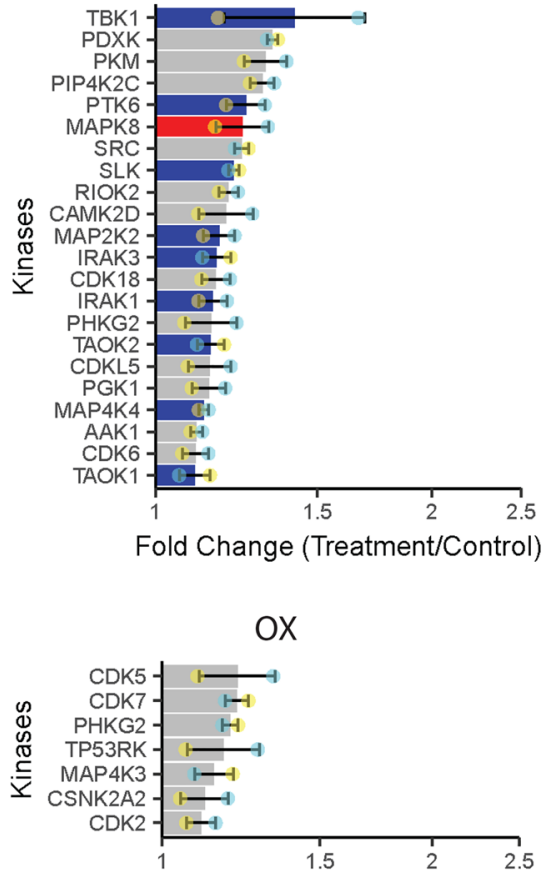

Fold Change (Treatment/Control)

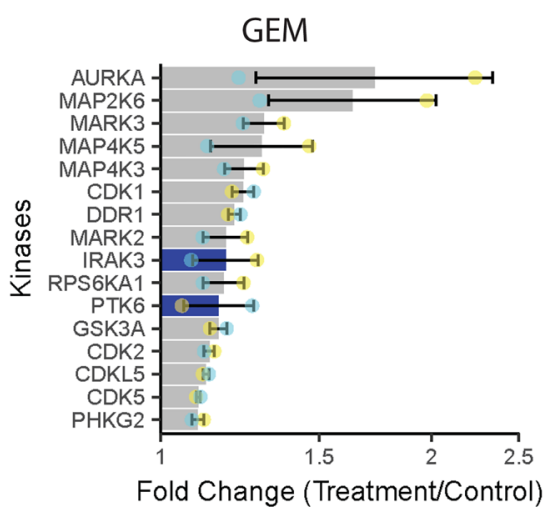

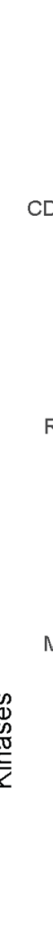

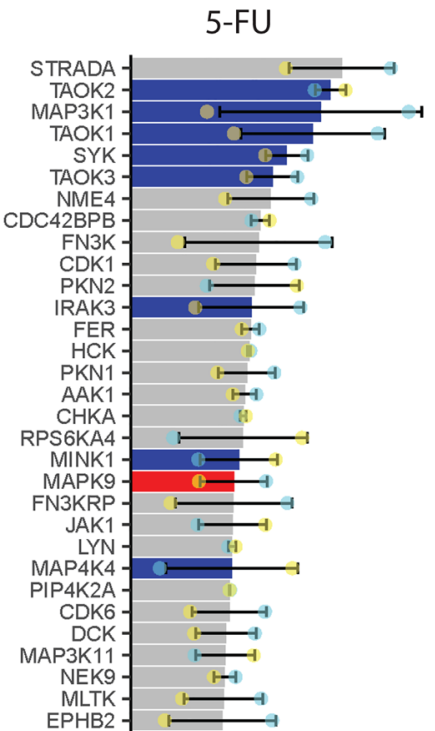

B
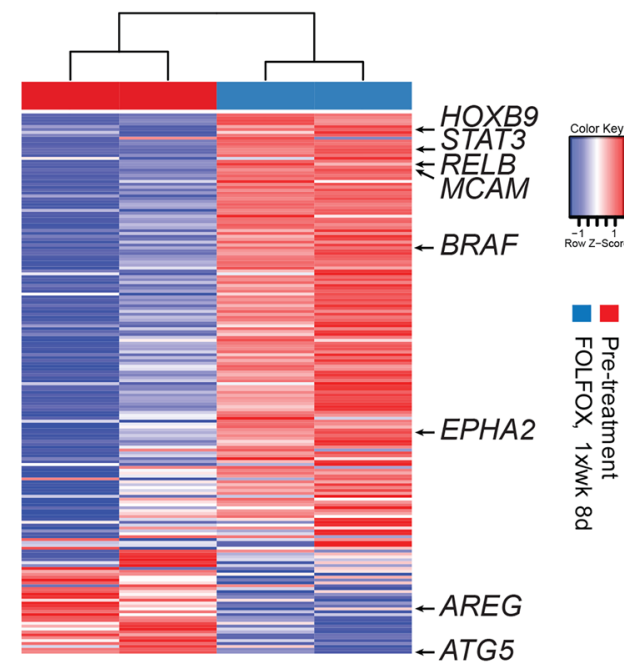

Pre-treat FOLFOX

C

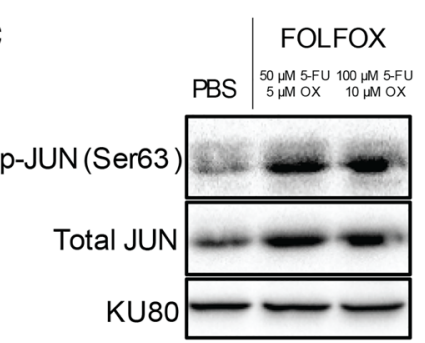

P411-T1

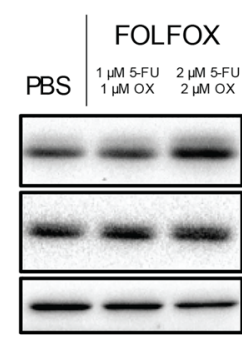

CFPAC-1

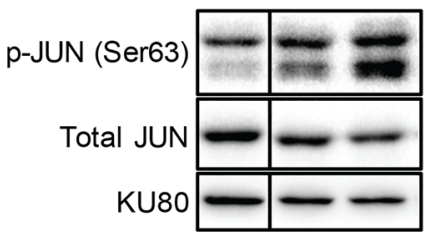

P422-T1

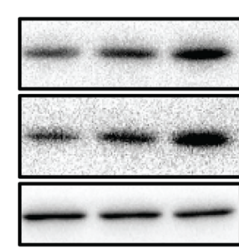

MIA PaCa-2

Figure 1. 5-FU and FOLFOX chemotherapy regimens induce druggable activation of JNK and JUN. (A) MIB-MS performed on PDX tumors biopsied before and after treatment with individual chemotherapy regimens (5-FU, 8-day [8d] $100 \mathrm{mg} / \mathrm{kg}$ 5-FU + LEU in P108-T1 PDX tumors; OX, 8d 5 mg/kg in P108-T1 PDX tumors; GEM, 3d 80 mg/kg in P108-T1 PDX tumors) or FOLFOX compared with vehicle-treated PDX tumors (3d $100 \mathrm{mg} / \mathrm{kg} 5-\mathrm{FU}+\mathrm{LEU}$ and $5 \mathrm{mg} / \mathrm{kg}$ OX in P319-T1 PDX tumors). JNK1 (MAPK8) and JNK2 (MAPK9) are shaded red, and kinases known to activate JNK are shaded blue. Biological replicate values are indicated by cyan and yellow dots $(n=2)$. (B) RNA-seq expression of predicted JUN transcriptional target genes (MSigDB: CREBP1CJUN_01) in FOLFOX-treated and matched pretreatment biopsy tumors, with labeled genes with known roles in cancer signaling. (C) Representative immunoblots showing upregulation of $p$-JUN after indicated doses of FOLFOX in PDX-derived lines P411-T1 and P422-T1, as well as ATCC cell lines CFPAC-1 and MIA PaCa-2. Vertical line indicates noncontiguous samples that were treated and collected simultaneously and run on the same gels. KU80 used as loading control.

As an orthogonal approach, we performed an unbiased compound library synergy screen in combination with FOLFOX in the P422-T1 PDX-derived cell line (PDX-CL) to identify druggable molecular targets for inhibition in combination with FOLFOX. Growth inhibition over 72 hours was quantified after FOLFOX alone and in combination with 176 kinase and other small molecule inhibitors by CellTiter-Glo, and hits were ranked by $\Delta$ Bliss analysis to reveal several compounds with synergy across a range of dose combinations (Table 1). The irreversible JNK inhibitor JNK-IN-8 demonstrated the second highest overall synergy with FOLFOX (16), while the nonspecific JNK inhibitor SP600125 ranked outside of the top 100 compounds with a positive $\Sigma \Delta$ Bliss score, indicating overall antagonism rather than synergy between the drugs.

To further evaluate JNK signaling following FOLFOX at the transcriptional level, we performed RNA sequencing (RNA-seq) on matched pretreatment biopsies and FOLFOX-treated P411-T1 PDX tumors. 
FOLFOX led to upregulation of expression of a curated set of predicted JUN transcription factor binding targets such as STAT3, BRAF, and EPHA2 $(40,41)$, linking the JNK pathway upregulation identified by MIB-MS to increased JUN transcription factor activity (Figure 1B). These results were validated in vitro with P411-T1 and P422-T1 PDX-CLs, as well as CFPAC-1 and MIA PaCa-2 established PDAC cell lines. All of these lines showed overexpression of phosphorylated JUN (p-JUN) and, in some cases, total JUN protein 12-48 hours after FOLFOX doses with minimal growth inhibitory effects (Figure 1C).

JNK-JUN inhibition with the highly specific irreversible inhibitor JNK-IN-8 is an attractive therapeutic strategy in PDAC. JNK-JUN overexpression has been observed in PDAC (28), but this overexpression has not been linked to differences in patient survival. Therefore, we examined the link between patient survival and expression of JNK1, JNK2, and JUN using data from 146 patients with primary PDAC in The Cancer Genome Atlas (TCGA) data set (42). High tumor expression of JNK1 and of the JUN signature shown to be upregulated by FOLFOX were associated with significantly shorter patient survival; in contrast, there was little association between JNK2 expression and patient survival (Figure 2A and Supplemental Figure 1; supplemental material available online with this article; https://doi.org/10.1172/jci.insight.129905DS1).

Our library compound screen linked the irreversible JNK inhibitor JNK-IN-8 — but not the well-studied nonspecific SP600125 inhibitor - to synergy with FOLFOX, prompting us to characterize the signaling effects and specificity of JNK-IN-8 in PDAC. Irreversible inhibitors like JNK-IN-8 permanently inhibit targets through formation of covalent bonds and are attractive biological tools and drug candidates due to their specificity and durable effects at relatively low doses (43). JNK-IN-8 led to strong decreases in p-JUN by 24 hours at $1 \mu \mathrm{M}$ JNK-IN-8, with complete disappearance of p-JUN at a higher $10 \mu \mathrm{M}$ JNK-IN-8 dose (Figure 2B). As reported previously, JNK-IN-8 covalent binding resulted in a complete and easily visible electrophoretic shift in the JNK1/2 protein population that allowed for direct visualization of inhibitor binding (Figure 2B) (16). JNK-IN-8 did not reduce JNK expression or decrease its ability to be phosphorylated by upstream activators; in fact, it led to a compensatory increase in p-JNK.

Next, we assessed the kinase binding profile of JNK-IN-8 using MIB-MS after a short 1-hour treatment with DMSO vehicle or $1 \mu \mathrm{M}$ JNK-IN-8. Decreases in levels of functional kinases by MIB-MS following drug treatment indicate inhibitor binding and/or kinase inactivation that prevent capture by bead-bound inhibitors and reduce detection by MS. Across 218 kinases that were detected in both samples, only JNK1 and JNK2 expression were considerably decreased by JNK-IN-8, indicating extremely high specificity (Figure 2C).

JUN transcription factor activity has been linked to invasive phenotypes (44), so we compared cell invasion through basement membrane matrix after low doses of JNK-IN-8 that knock down p-JUN but do not affect cell viability, revealing dose-dependent decreases in invasion after JNK-IN-8 (Figure 2D).

JNK-IN-8 enhances FOLFOX growth inhibition and reverses FOLFOX-induced JUN activation in vitro. Evaluation of JNK-IN-8 in PDAC is still limited to well-characterized cell lines, and JNK-IN-8 has not been studied in combination with chemotherapies (30). Therefore, we assessed the therapeutic potential of JNK-IN-8 in combination with FOLFOX and its components in vitro with 72-hour 3-(4,5-dimethylthiazol-2-yl)-2,5-diphenyl tetrazolium bromide (MTT) assays across a wide range of constant-ratio dose combinations (Figure 3A), as well as with longer-term 14-day colony formation assays dosed with drugs every 3 days and visualized by crystal violet stain (Figure 3B). In both 3-day and 14-day growth assays, there was striking enhancement of growth inhibition following treatment with combinations of FOLFOX and JNKIN-8 compared with individual drugs, especially in P411-T1 and CFPAC-1, while there was less robust synergy in P422-T1 and less still in MIA PaCa-2. Interestingly, there was no relationship between the strength of synergy and sensitivity to FOLFOX or JNK-IN-8 alone, with CFPAC-1 and MIA PaCa-2 being the most sensitive to FOLFOX and JNK-IN-8 individually but having the most divergent synergy profiles.

We then evaluated corresponding changes in p-JUN expression after each treatment, observing complete reversal of FOLFOX-mediated p-JUN activation with the addition of JNK-IN-8 across all lines (Figure 3C). Synergy appeared to be correlated with activation of p-JUN expression following FOLFOX alone, with MIA PaCa-2 showing the weakest synergy and the least upregulation of p-JUN after FOLFOX, while CFPAC-1 displayed both the most synergy and the strongest FOLFOX-induced activation of p-JUN (Figure 3D). In all lines, synergy was observed across a wide range of doses, as quantified by the combination index method (Figure $3 \mathrm{E}$ and Supplemental Table 1) (45). Notably, synergy between FOLFOX and JNK-IN-8 was driven by 5-FU, while viability after OX in P411-T1 was unaffected by the addition of JNK-IN-8 (Supplemental Figure 2).

JNK-IN-8 effectively inhibits JUN in organoids and in vivo to enhance FOLFOX growth inhibition. There continues to be an urgent need for new approaches to PDAC treatment, given the still-limited options available to patients. 
Table 1. Top-ranked synergistic drug combinations in a synergy screen between FOLFOX and 176 small compounds.

\begin{tabular}{cccc}
\hline Rank & Compound & Target(s) & $\boldsymbol{\Sigma} \Delta$ Bliss \\
\hline 1 & THZ1 & CDK7 & -0.818 \\
\hline $\mathbf{2}$ & JNK-IN-8 & JNK & -0.722 \\
\hline 3 & CSK-J4 & JMJD3/UTX & -0.660 \\
\hline 4 & CDK9 inhibitor & CDK9 & -0.593 \\
\hline 5 & AT7519 & CDK9/CDK5 & -0.549 \\
\hline 6 & PF-3758309 & PAK4/1/6/5 & -0.494 \\
\hline 7 & Flavopiridol HCI & CDK1/2/4/6 & -0.402 \\
\hline 8 & Dinaciclib (SCH727965) & CDK1/2/5/9 & -0.402 \\
\hline 9 & CHIR-99021 (CT99021) HCI & CSK-3 & -0.396 \\
\hline 10 & MK-8776 (SCH 900776) & CHEK1 & -0.374 \\
\hline$\cdot$ & $\cdot$ & $\cdot$ & $\cdot$ \\
\hline $\mathbf{1 0 7}$ & $\cdot$ & $\cdot$ &. \\
\hline $\mathbf{1 0 7}$ & $\mathbf{S P 6 0 1 2 5}$ & JNK & 0.565 \\
\hline
\end{tabular}

P422-T1 PDX-derived cells treated for 72 hours and growth was assessed by CellTiter-Glo relative to DMSO controls. Synergy score calculated with Bliss independence modeling, where negative $\Sigma \Delta$ Bliss scores indicate synergy.

JNK-IN-8 is from the emerging class of covalent, irreversible inhibitors and has not yet been studied in 3D organoid cultures or in vivo models of PDAC that are thought to be representative of patient tumor biology and treatment responses $(46,47)$. Therefore, we evaluated JNK-IN-8 in combination with FOLFOX in PDX-derived P319-T1 organoids and in 4 PDX or cell line xenograft tumors in NOD SCID $\gamma$ (NSG) immunodeficient mice.

Organoids were generated from dissociated PDX tumors in suspensions of basement membrane matrix and were then treated for 5 days with a range of doses of FOLFOX, JNK-IN-8, and the combination. Organoid growth was markedly stunted by the combination as compared with either individual drug, producing strong synergy at low doses at which neither individual drug affected growth (Figure 4A).

S.c. implanted PDX tumors were biopsied for pretreatment control samples, and then mice were randomly assigned to be treated for 28 days in 4 groups: saline, JNK-IN-8 (30 mg/kg, 2×/week), FOLFOX (50:5:100 $\mathrm{mg} / \mathrm{kg} 5$-FU/OX/LEU, $1 \times /$ week), and the combination of JNK-IN-8 and FOLFOX. FOLFOX dosing was chosen to mirror clinical pharmacokinetics while not approaching previously determined maximum-tolerated doses in mice (48). I.p. dosing of JNK-IN-8 (30 mg/kg, $2 \times /$ week) with and without FOLFOX was tolerated without weight loss or significant toxicity in tumor-bearing mice from all lines tested and led to strong inhibition of tumor p-JUN expression after 8-day treatment courses (Supplemental Figures 3, A and B).

Consistent with in vitro data, JNK-IN-8 in combination with FOLFOX led to p-JUN inhibition and enhanced tumor growth inhibition to varying degrees in combination with FOLFOX in P319-T1, CFPAC-1, and MIA PaCa-2 xenograft tumors (Figure 4B and Supplemental Figure 3C). Interestingly, addition of JNK-IN-8 to FOLFOX did not consistently inhibit tumor growth in P411-T1, a line with strong in vitro synergy (Figure 4C). This lack of tumor growth response corresponded to a lack of sustained p-JUN inhibition in these tumors after 28-day treatment with JNK-IN-8 alone and in combination with FOLFOX (Supplemental Figure 4). In aggregate, the combination of FOLFOX and JNK-IN-8 led to stabilization of tumor growth near the end of the study period across lines, with week-over-week shrinkage in many tumors and marked regression in one P319-T1 tumor, suggesting that continuing treatment beyond 28 days might further enhance synergy (Supplemental Figure 3D).

Synergy between FOLFOX and JNK-IN-8 is linked to FOLFOX-mediated DNA damage and cell cycle responses. Chemosensitivity to antineoplastic agents like 5-FU is often facilitated by failure to sustain ongoing DNA replication and progression through S-phase of the cell cycle. Therefore, we assessed whether the varying strengths of synergy between FOLFOX and JNK-IN-8 observed across lines was related to altered DNA damage response and cell cycle dysregulation. Cells were treated for 72 hours with doses chosen such that the individual drugs caused minimal growth inhibition, no synergy occurred with the combination in MIA PaCa-2, and strong synergy was observed in the other 3 lines (Figure 3A). Propidium iodide (PI) staining and flow cytometry of these treated cells revealed visible S-phase arrest after FOLFOX in P411-T1, 
A
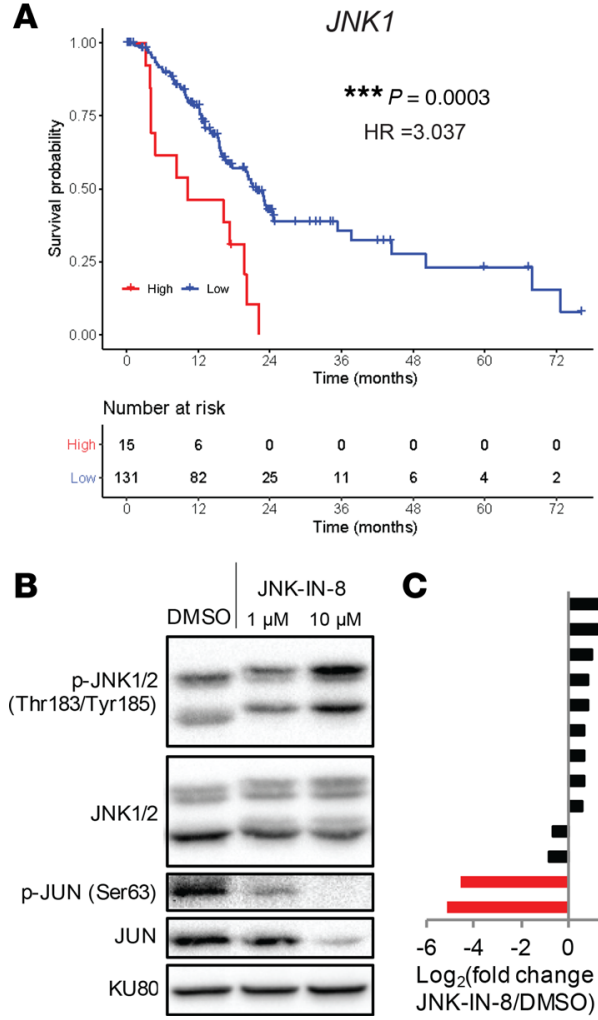
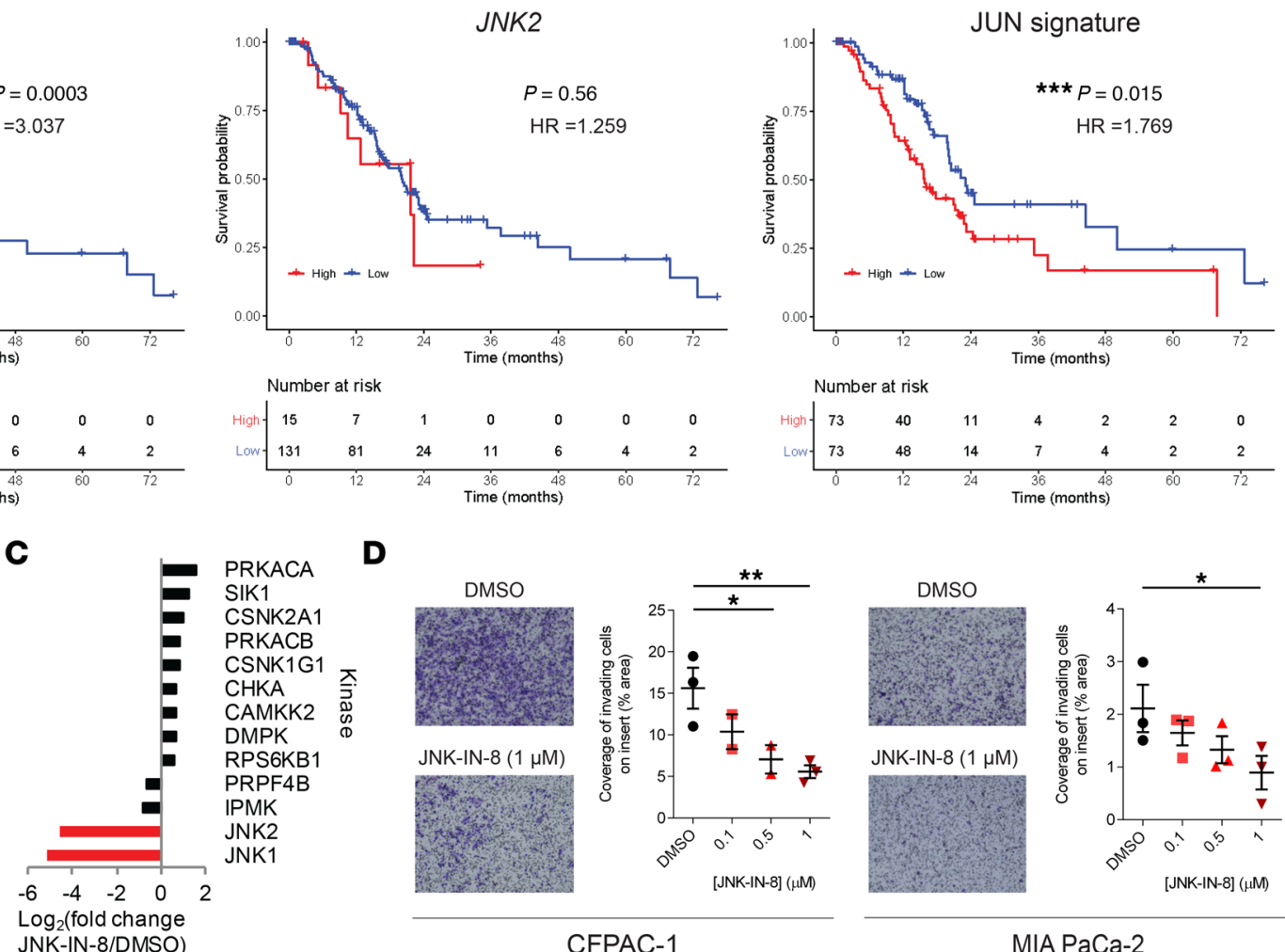

D
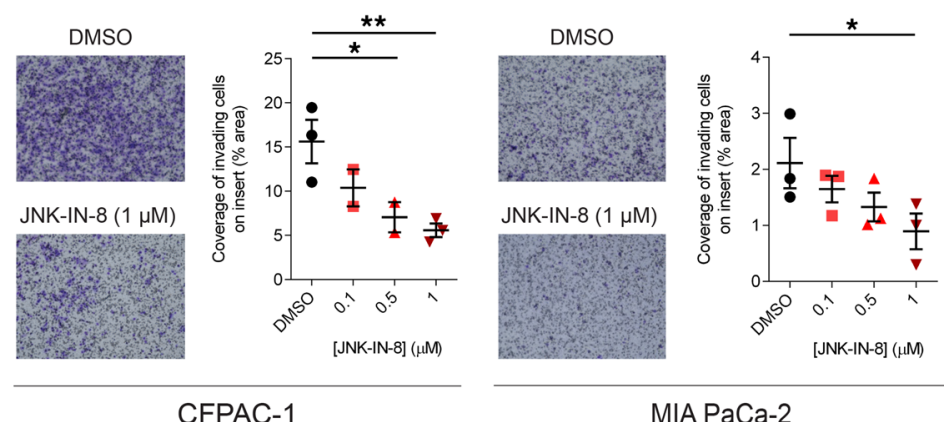

MIA PaCa-2

Figure 2. JNK-JUN inhibition with the highly specific irreversible inhibitor JNK-IN-8 is an attractive therapeutic strategy in PDAC. (A) Kaplan-Meier plots comparing survival of patients with resected PDAC from the TCGA RNA-seq data set after splitting the cohort by expression of JNK1 or JNK2, or by the mean of rankings of 257 predicted JUN transcriptional targets (MSigDB: CREBP1CJUN_01). Significance determined by log-rank test shown with $P$ values and hazard ratios (HR) determined by Cox proportional-hazards model. (B) Representative immunoblot after 12-hour treatment with DMSO or the covalent JNK inhibitor JNK-IN-8 in a P411-T1 PDX-derived cell line. KU80 used as loading control $(n=2)$. (C) MIB-MS analysis 1 hour after $1 \mu \mathrm{M} \mathrm{JNK-IN-8} \mathrm{treatment} \mathrm{in}$ CFPAC-1 cells relative to DMSO-treated controls. JNK1 and JNK2 are highlighted in red. A total of 218 kinases were detected in both treatment and control; only kinases with $\mid$ fold change $>1.5$ are shown. (D) Invasion through Matrigel transwells with representative images (original magnification $\times 20$ [ $1 \times 20]$ ) and quantified growth analyzed by 1-way ANOVA with Holm-Sidak's multiple comparisons test $(n=2-3)$. ${ }^{*} P<0.05 ;{ }^{* *} P<0.01,{ }^{* * *} P<0.001$.

P422-T1, and CFPAC-1, but not in MIA PaCa-2 (Figure 5A). MIA PaCa-2 cells treated with JNK-IN-8 alone and in combination with FOLFOX displayed an increased population of cells in S-phase, while little change occurred with JNK-IN-8 in the other 3 lines, suggesting that JNK-IN-8 facilitates ongoing replication and S-phase progression in MIA PaCa-2, even in the face of FOLFOX treatment.

To examine transcriptional changes in JUN activity and DNA damage pathways, we performed RNAseq on CFPAC-1 and MIA PaCa-2 cells treated for 72 hours with vehicle, a low FOLFOX dose, a moderate FOLFOX dose, and combinations of both FOLFOX doses with $1 \mu \mathrm{M}$ JNK-IN-8. Consistent with these lines' disparate strengths of JUN activation following FOLFOX and opposing effects of JNK and JUN overexpression on sensitivity to FOLFOX (Figure 3, C and E; Figure 5; and Supplemental Figure 5), FOLFOX led to strong, dose-dependent upregulation of a curated set of predicted JUN targets in CFPAC-1, but not in MIA PaCa-2 (Figure 5B) $(40,41)$. We next took an unbiased approach to isolating other pathways that were differentially affected by the combination of FOLFOX and JNK-IN-8 between CFPAC-1 and MIA PaCa-2 using the DESeq2 program (49). These genes were functionally classified using Gene Set Enrichment Analysis (GSEA) (41), revealing the base excision repair (BER) DNA damage pathway as one of the most highly differentially expressed gene sets (adjusted $P=1.77 \times 10^{8}$ ) (Molecular Signatures Data base ID [MSigDB]: DACOSTA_UV_RESPONSE_VIA_ERCC3_UP) (40). In MIA PaCa-2, JNKIN-8 caused marked reversal in FOLFOX-induced downregulation of many of these base excision repair (BER) related genes known to promote survival after DNA damage (Figure 5C). Conversely, in CFPAC-1, both FOLFOX and the combination resulted in decreased expression of many of these genes at a strongly synergistic dose combination. High BER pathway activity has been associated with poor patient outcomes and resistance to both 5-FU and platinum chemotherapies (50), and inhibitors of this pathway have been 
A

P411-T1

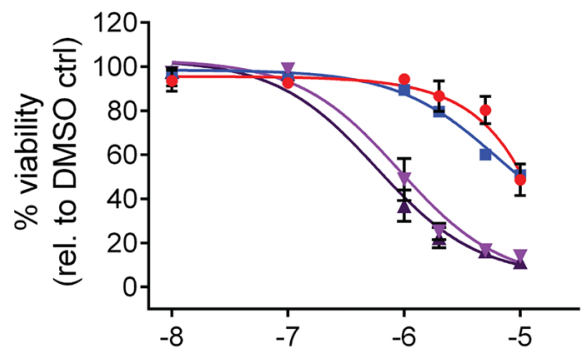

P422-T1

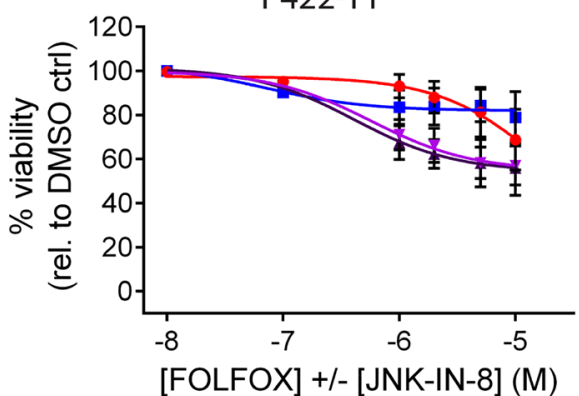

$[$ FOLFOX] +/- [JNK-IN-8] (M)
CFPAC-1
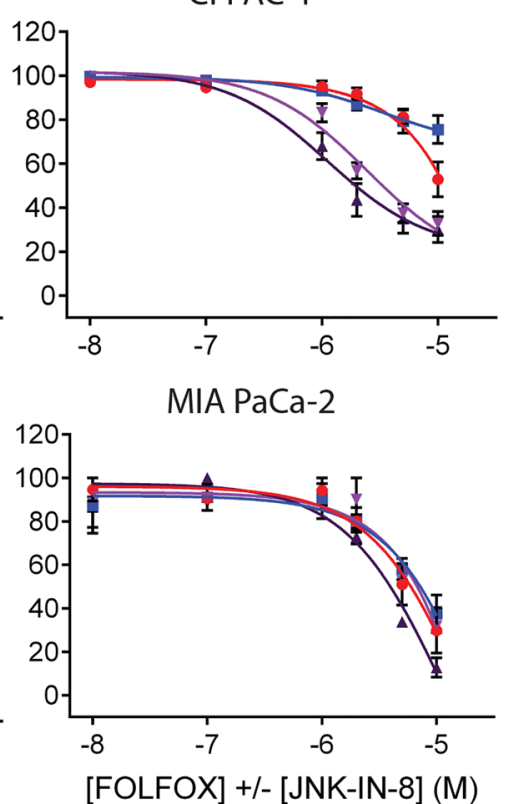

$\rightarrow$ JNK-IN-8 $\rightarrow$ FOLFOX $\rightarrow$ COMBO 10:1:5 $\rightarrow$ COMBO 10:1:10

B

P411-T1

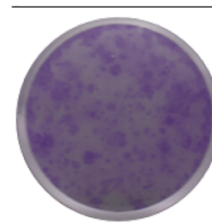

DMSO

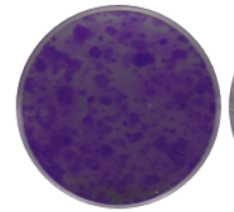

JNK-IN-8

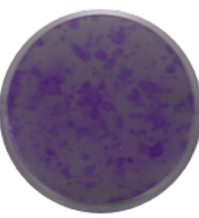

FOLFOX

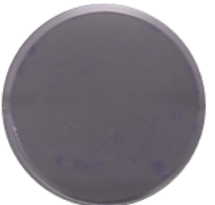

FOLFOX+ JNK-IN-8

P422-T1

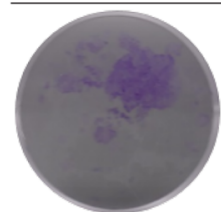

DMSO

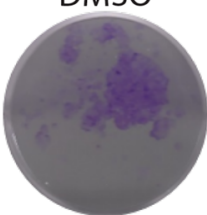

JNK-IN-8

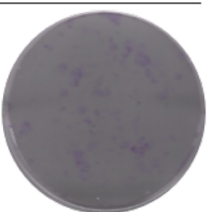

FOLFOX

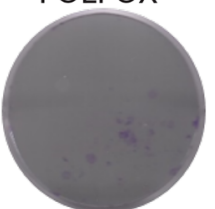

FOLFOX+ JNK-IN-8
CFPAC-1

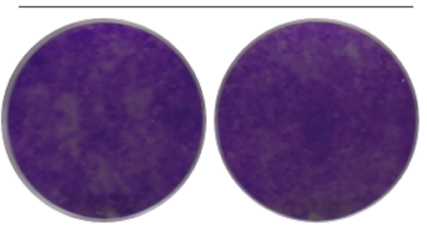

FOLFOX

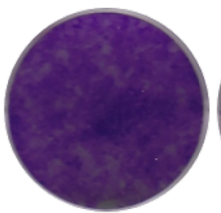

JNK-IN-8

MIA PaCa-2

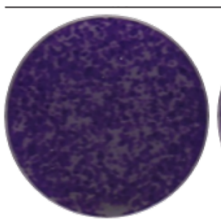

DMSO

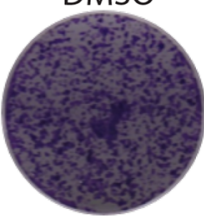

JNK-IN-8

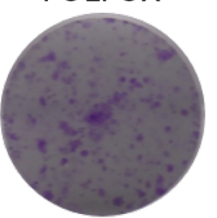

FOLFOX+ JNK-IN-8

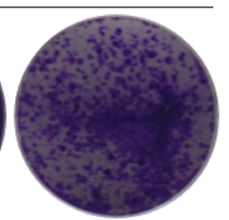

FOLFOX

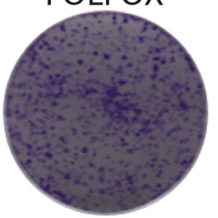

FOLFOX+ JNK-IN-8
C JNK-IN-8 $-+-\quad+\quad+$

p-JUN (Ser63)

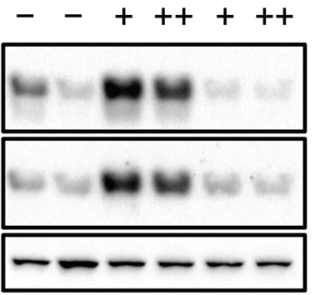

P411-T1

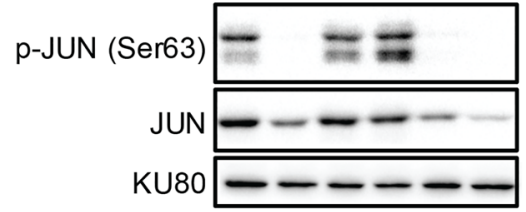

$\mathrm{P} 422-\mathrm{T} 1$

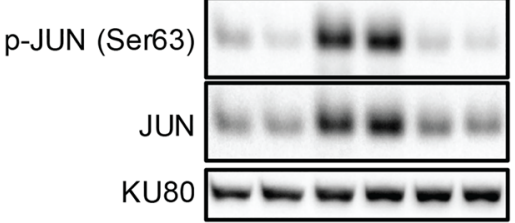

CFPAC-1

p-JUN (Ser63)

JUN KU80

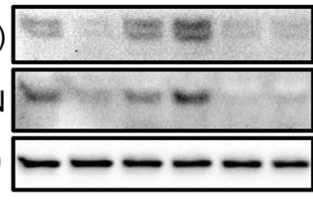

MIA PaCa-2

D

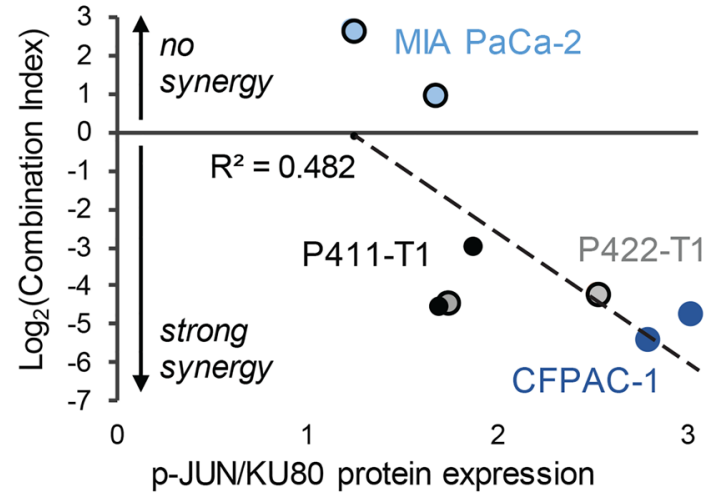

E

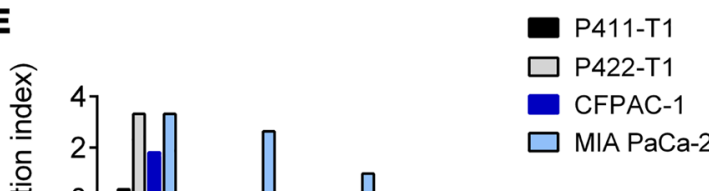


Figure 3. JNK-IN-8 enhances FOLFOX growth inhibition and reverses FOLFOX-induced JUN activation in vitro. (A) Dose-response curves for JNK-IN-8 (red), FOLFOX (blue), and 2 constant ratio combinations of the pair (purples). Results shown are mean \pm SEM normalized percent viability ( $n=3$ ). (B) Crystal violet-stained cell colonies after 14 days of twice-weekly dosing of the indicated drug regimen (5-FU/OX/JNK-IN-8 [nM]: P411-T1, 400:40:1000; P422-T1, 200:20:200; CFPAC-1, 200:20:200; MIA PaCa-2, 1000:100:1000). (C) Representative immunoblots for P411-T1, P422-T1, CFPAC-1, and MIA PaCa-2 cells treated for 72 hours with the designated regimen (JNK-IN-8 $=1 \mu \mathrm{M} ;+$ FOLFOX $=I_{10}$ dose by MTT; ++FOLFOX $=I_{25}$ dose by MTT). KU80 was used as loading control for 422-T1, run on parallel gel. (D) Synergy represented by $\log _{2}(\mathrm{Cl})$ plotted against $\mathrm{p}-\mathrm{JUN} / \mathrm{KU} 80$ immunoblot expression at 1- and $2-\mu \mathrm{M}$ doses. Trendline with correlation coefficient $\mathrm{R}^{2}$ shown. (E) Synergy as $\log _{2}(\mathrm{Cl})$ across doses from 72-hour MTT assay shown in $\mathbf{A}$, where negative $\log _{2}(\mathrm{Cl})$ values indicate synergy.

evaluated in PDAC (51). Thus, JNK-IN-8-mediated BER activation in MIA PaCa-2 may prevent synergy from occurring in this line, despite decreased JUN activity, and BER inhibition could represent a previously undescribed mechanism for resistance to JNK inhibition.

Finally, we examined mediators of DNA damage response with immunoblots in these treated CFPAC-1 and MIA PaCa-2 cells following cell fractionation into cytosolic and chromatin-bound fractions, revealing a robust DNA damage response after FOLFOX in CFPAC-1 but not in MIA PaCa-2 (Figure 5D). p-CHK1, a marker for the active response to replication stalling and DNA damage caused by cytotoxic drugs like 5-FU, was strongly upregulated at every time point by FOLFOX and the combination in CFPAC-1, but it was completely unaffected in MIA PaCa-2. Similarly, the same expression pattern was found for ubiquitinated FANCD2, which is produced in response to DNA cross-linking by platinum chemotherapies like OX and other DNA damage. These responses support the cell cycle profiles showing profound S-phase accumulation after FOLFOX in CFPAC-1 without corresponding changes in MIA PaCa-2. Despite a known role for JNK in activating double-stranded break (DSB) repair (52, 53), JNK inhibition with and without FOLFOX led to only gradual accumulation of DSBs, as indicated by late expression of p-ATM, a marker for DNA DSBs, and no change in the ATM effector p-CHK2. Taken together, these results suggest that JUN activation, DNA damage response, and cell cycle dysregulation following FOLFOX may each be critical for facilitating synergy when combined with JNK-IN-8.

JUN is necessary for facilitating resistance to FOLFOX and for maintaining sensitivity to JNK-IN-8. Thus far, our results indicated that JUN contributes to resistance to FOLFOX and sensitivity to JNK-IN-8, but JNK is also known to activate several transcription factors in order to carry out its myriad intracellular functions (17-19). Therefore, to isolate the role of JUN in mediating drug effects, we compared cell growth after treatment with FOLFOX and JNK-IN-8 in CFPAC-1 cells following lentiviral-mediated transduction of shRNA directed against $J U N(\operatorname{sh} J U N)$ compared with CFPAC-1 cells transduced with the corresponding shRNA empty vector (shEV). While residual levels of activated p-JUN and total JUN remained after shRNA transduction (Figure 6A), JUN knockdown led to reproducibly increased sensitivity to FOLFOX at all experimental doses, indicating that JUN is necessary for facilitating resistance to FOLFOX in this line (Figure 6B). Moreover, JUN knockdown consistently promoted resistance to JNK-IN-8, especially at low doses found to lead to the greatest synergy when JNK-IN-8 was combined with FOLFOX (Figure 3), confirming that JUN inhibition is responsible for at least part of the cytotoxic effects of JNK-IN-8 (Figure 6C).

JUN and JNK1 are sufficient to induce FOLFOX resistance and abrogate synergy between FOLFOX and $J N K-I N-8$. The individual roles for JNK1 and JNK2 have not been separated in PDAC, but high JNK1 expression was more strongly associated with shorter patient survival than high JNK2 expression (Figure 2A). Therefore, we sought to investigate the distinct contributions of JNK1, JNK2, and JUN in mediating sensitivity to FOLFOX and synergy with JNK-IN-8 through genetic manipulation of CFPAC-1 and MIA PaCa-2, cell lines with strong and weak in vitro synergy, respectively (Figure 3).

Lentiviral-mediated plasmid transduction was carried out to allow for doxycycline-inducible expression (dox-inducible expression) of a GFP-tagged EV (EV-pCW57-GFP) cloned to contain individual JUN and $J N K$ constructs with varying protein activation states: (a) WT JUN, JNK1, and JNK2 transgenes (JUN and JNK1/2-WT); (b) constitutively active (CA) JNK1 or JNK2 fused to the upstream MAP2K7 (JNK1/2CA); and (c) phosphorylation dead (PD) JNK1 or JNK2 that are unable to be activated due to T183/ Y185A183/F185 mutations in the critical dual phosphorylation site (JNK1/2-PD) (17, 21). We confirmed that treatment with $1 \mu \mathrm{g} / \mu \mathrm{L}$ dox for as little as 4 hours led to expression of GFP by microscopy (Figure 7A) and of JUN transgene without expression in the absence of dox or under any conditions in the EV-infected line (Figure 7B). As expected, induction of JNK1-WT and JNK2-WT expression was not accompanied by increased p-JNK or p-JUN activation, while JNK1-CA and JNK2-CA led to rapid upregulation of both p-JNK and p-JUN (Figure 7, C and D, and Supplemental Figure 5A). Importantly, JNK1-CA led to visibly 
A

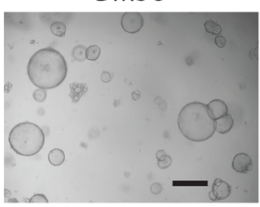

FOLFOX

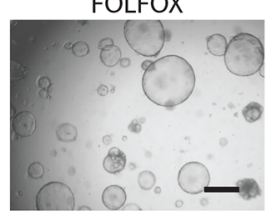

JNK-IN-8

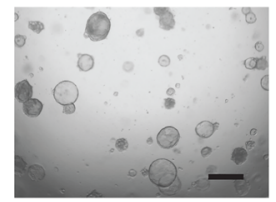

FOLFOX+JNK-IN-8

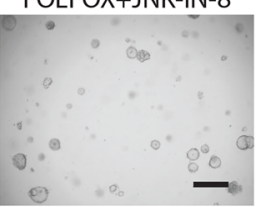

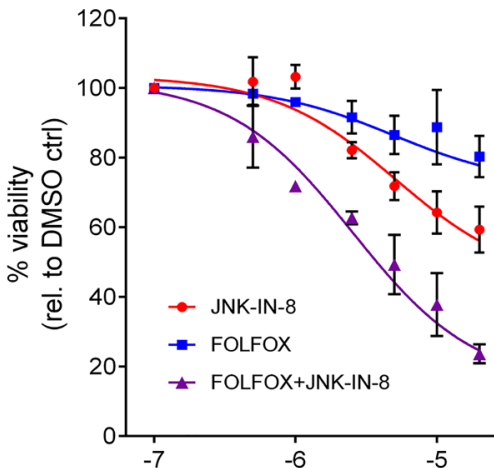

$[$ FOLFOX] +/- [JNK-IN-8] (M)
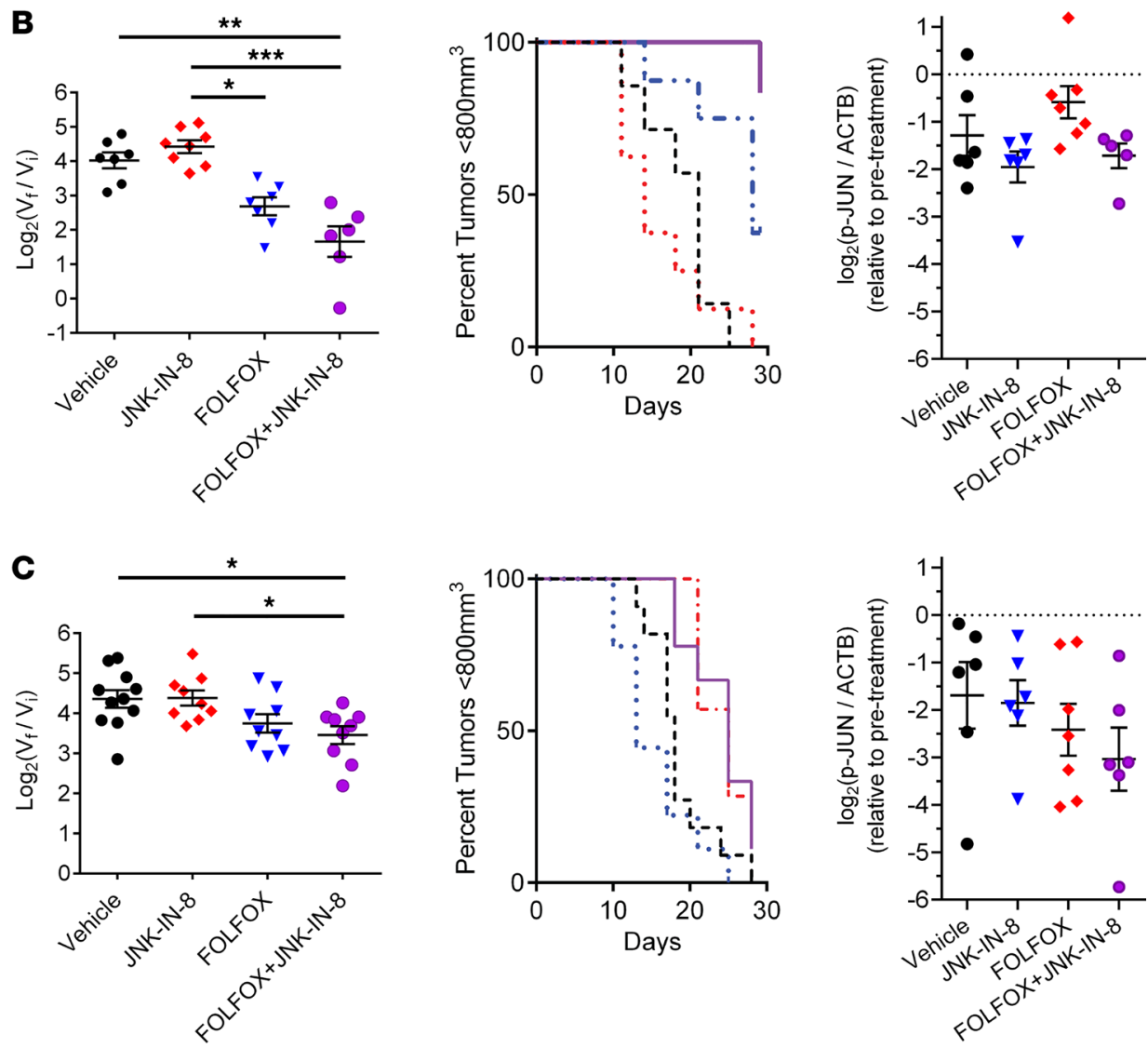

Vehicle

JNK-IN-8

FOLFOX

FOLFOX+JNK-IN-8

Figure 4. JNK-IN-8 enhances growth inhibition by FOLFOX in PDX organoids and tumors corresponding to sustained p-JUN inhibition. (A) Organoid viability by bright-field microscopy at $1-\mu \mathrm{M}$ doses of indicated treatments (left) and quantified across a broad range of doses by CellTiter-Glo 3D with normalization to DMSO vehicle (right) in 319-T1 organoids $(n=2)$. Scale bars: $500 \mu \mathrm{m}$. (B) In vivo 319-T1 PDX tumor growth displayed as box plots of $\log _{2}$ (tumor volume fold change at $28 \mathrm{~d}$ endpoint versus treatment initiation) and Kaplan-Meier survival plots with a tumor volume cutoff of $800 \mathrm{~mm}^{3}$. Log transformed ratio of p-JUN/ACTB protein levels by immunoblot from the same 319-T1 PDX tumors harvested before and after $28 \mathrm{~d}$ treatments of respective drug regimens. Data normalized to pretreatment biopsy tumors. ACTB used as loading control. (C) In vivo 411-T1 PDX tumor growth data and p-JUN inhibition by immunoblot as above. Each group contained at least 5 mice. Significance determined by 1-way ANOVA with Tukey multiple comparisons tests. ${ }^{*} P<0.05$; ${ }^{*} P<0.01,{ }^{* *} P<0.001$. 

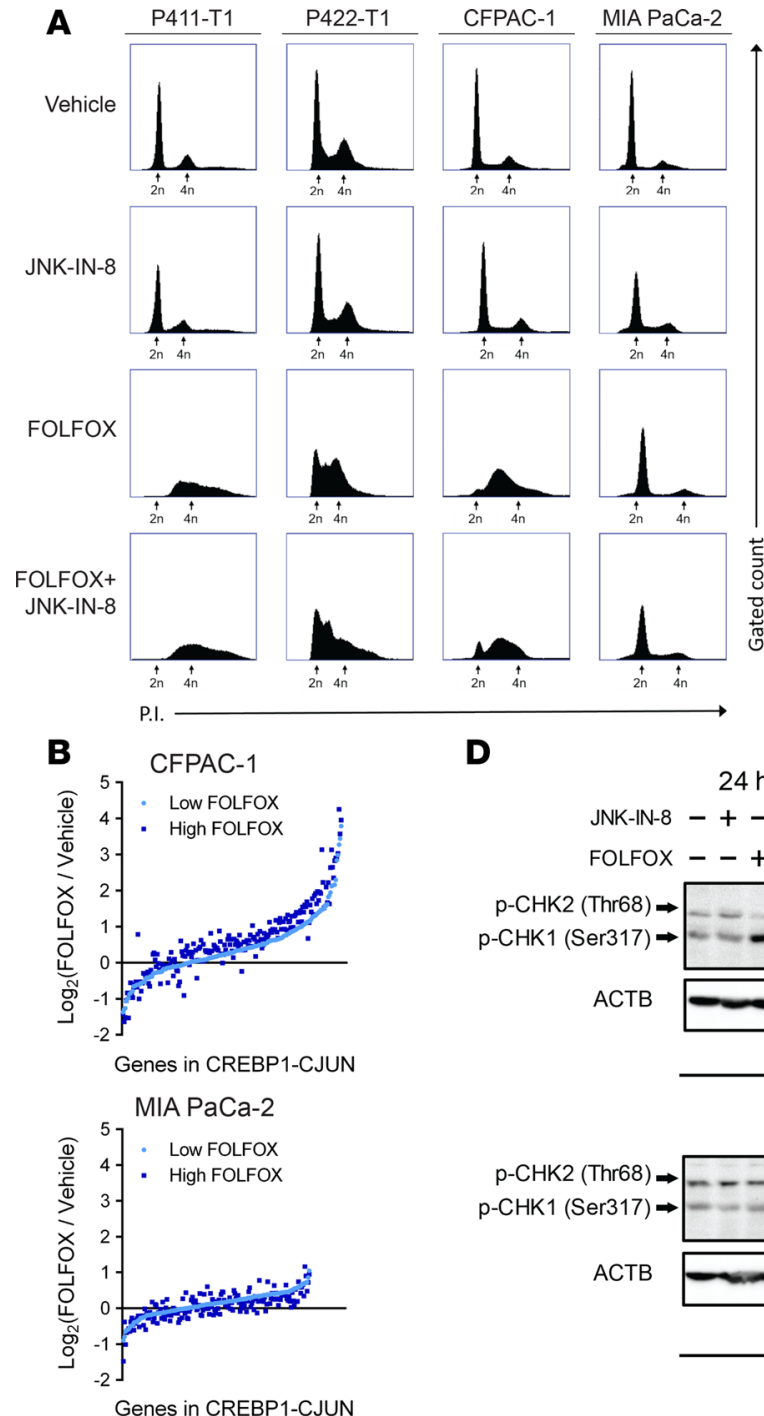

D
C
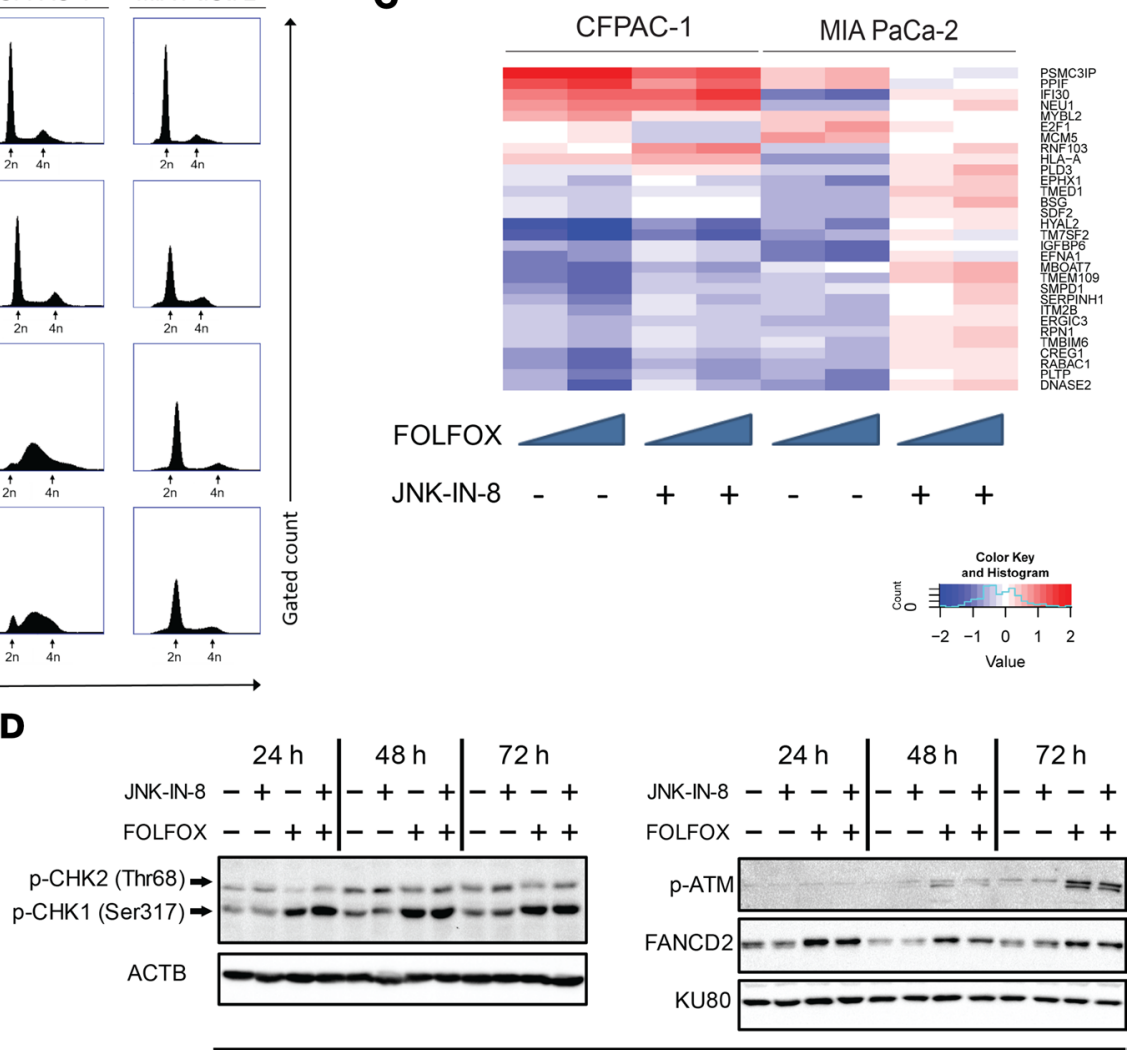

CFPAC-1
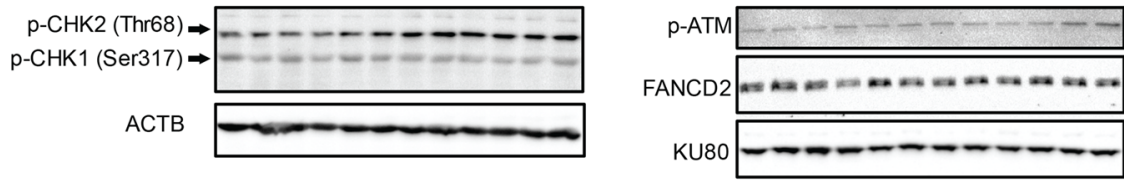

MIAPaCa-2

Figure 5. Synergy between FOLFOX and JNK-IN-8 may be facilitated by cell cycle dysregulation and DNA damage response. (A) Representative flow cytometry assessing PI staining ( $x$ axes) after indicated treatments in each cell line $(n=2)$. At least 50,000 gated events were recorded per condition ( $y$ axes) $(n=3)$. FOLFOX, $1 \mu \mathrm{M}$ 5-FU + 0.1 $\mu \mathrm{M}$ OX + $10 \mu \mathrm{M}$ LEU; JNK-IN-8, $1 \mu \mathrm{M}$. (B) RNA-seq expression of predicted JUN transcription factor targets (MSigDB: CREBP1CJUN_01) after 2 doses of FOLFOX normalized to DMSO vehicle in CFPAC-1 and MIA PaCa-2 cells. Each gene with mean expression greater than 1 transcript per million total transcripts (TPM) is represented by a single dot for low FOLOX (light blue) and high FOLFOX (dark blue). (C) RNA-seq expression of base excision repair (BER) DNA damage pathway genes in CFPAC-1 and MIA PaCa-2 (MSigDB: DACOSTA_UV_RESPONSE_VIA_ERCC3_UP), as identified by DESeq2 analysis for differentially expressed genes between lines in individual drug and combination treatments. (D) Immunoblots assessing DNA damage response genes 24,48 , and 72 hours after indicated treatments. KU 80 and ACTB were used as loading controls.

higher expression of activated p-JUN than did expression of JNK2-CA with similar expression of total JNK1-CA versus JNK2-CA, suggesting that JNK1 more robustly activates JUN.

Next, the effect of expression of these transgenes on sensitivity to FOLFOX and synergy between FOLFOX and JNK-IN-8 was assessed by MTT assays. Both dox-induced and no-dox control cells were seeded from a single population of plasmid-transduced cell lines that had not been exposed to dox, allowing for quantitative comparisons between growth in transgene expressing and control cells at each dose. After cells adhered, half were treated with $1 \mu \mathrm{g} / \mathrm{mL}$ dox for 8 hours to allow for complete induction of transgene protein expression; then, cells were treated with FOLFOX and/or JNK-IN-8 for 72 hours, followed by quantification of viability by MTT assay.

Expression of active JUN significantly increased resistance to FOLFOX and reduced synergy between FOLFOX and JNK-IN-8 (Figure 7B). Similarly, inducible expression of JNK1-CA, but not JNK1-WT or JNK1-PD, resulted in reduced sensitivity to FOLFOX and synergy with FOLFOX plus JNK-IN-8 (Figure 7C). JNK1-PD appeared to increase sensitivity to FOLFOX with and without JNK-IN-8, highlighting 
A
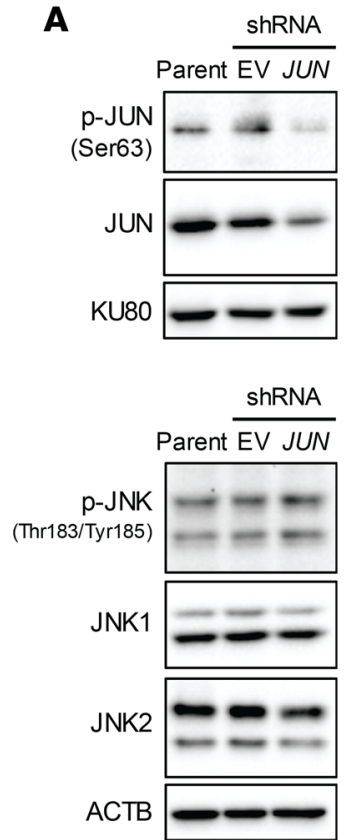

B

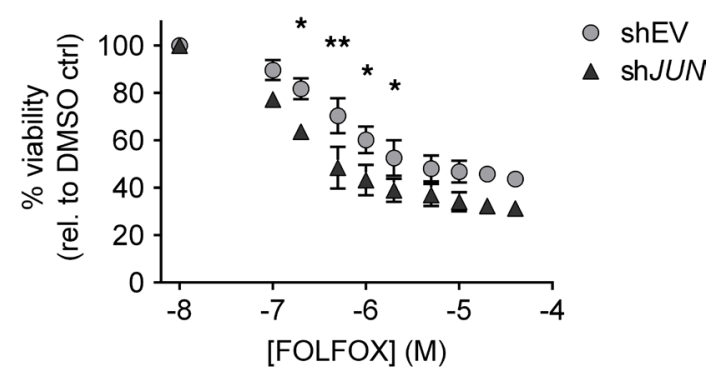

C

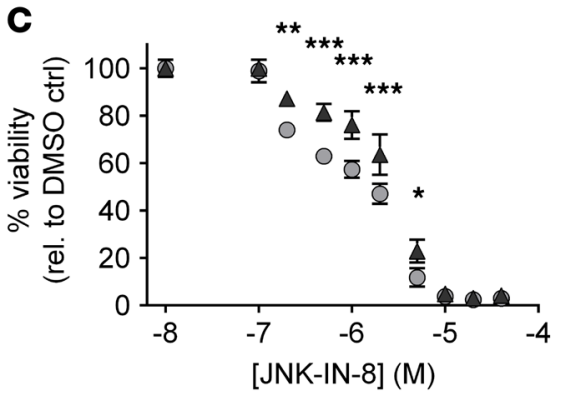

Figure 6. JUN is necessary for facilitating resistance to FOLFOX and for maintaining sensitivity to JNK-IN-8. (A) Immunoblots in parental CFPAC-1 and CFPAC-1 cells transduced with shJUN or shEV. KU80 and ACTB shown as loading controls. (B) FOLFOX dose-response curves in CFPAC-1 cell lines transduced with shJUN (triangles) or shEV (circles) and analyzed by 2-way ANOVA with Fisher's multiple comparison's test (mean \pm SEM shown, $n=3$ ). (C) JNK-IN-8 dose-response curves in the same 2 shRNA-transduced CFPAC-1 lines. ${ }^{*} P<0.05,{ }^{* *} P<0.01,{ }^{* *} P<0.001$.

the necessity of JNK phosphorylation for these effects and suggesting that this mutant JNK1 possesses dominant negative functions. In contrast to JNK1, JNK2 did not exert these strong effects, with JNK2-CA only modestly increasing resistance to FOLFOX and having no effect on synergy between FOLFOX and JNK-IN-8 (Figure 7D). These same induction experiments were also performed in MIA PaCa-2, cells with limited synergy between FOLFOX and JNK-IN-8. In this line, JUN and JNK1 did not lead to changes in sensitivity to FOLFOX or synergy with JNK-IN-8, further supporting that MIA PaCa-2 is less reliant upon JNK-JUN signaling in response to FOLFOX (Supplemental Figure 5B).

\section{Discussion}

Chemotherapies and chemotherapy combinations are widely used in the treatment of PDAC and almost all malignancies, but insufficient consideration has been placed on the signaling responses that underlie intrinsic tumor resistance to these treatments. In this study, we took parallel, unbiased approaches in patient-derived models of PDAC to assess specific kinase signaling pathways that facilitate resistance to 5-FU and FOLFOX.

Global changes in the functional kinome between matched pre- and posttreatment tumors were evaluated by MIB-MS kinome profiling, revealing upregulation of the JNK1-JNK2 signaling cascades after treatment with 5-FU and FOLFOX that did not occur after other chemotherapy regimens. Similarly, a synergy screen assessing growth inhibition after a compound library and FOLFOX prompted us to study the irreversible JNK inhibitor JNK-IN-8 for its ability to enhance FOLFOX efficacy. Together, these screens and our confirmatory experiments show that JNK activation by FOLFOX meaningfully contributes to prosurvival signaling that can be exploited by specific JNK inhibition.

Covalent inhibitors like JNK-IN-8 are steadily being developed, but evaluation of their biological effects in different tissues and cancers lags far behind. These inhibitors are attractive experimental tools and potential drugs because they result in rapid, permanent inhibition of target proteins, and - as we demonstrated for JNK-IN-8 - they can exhibit incredibly high specificity that stands in stark contrast to the nonspecific effects of reversible inhibitors like SP600125 long used to interrogate JNK functions (16, 43). In PDAC, JNK-IN-8 has been studied sparingly to validate SP600125 effects in combination with TNF-related apoptosis-inducing ligand (TRAIL) therapy (30) but, until now, had not been examined in combination with chemotherapies. 
A

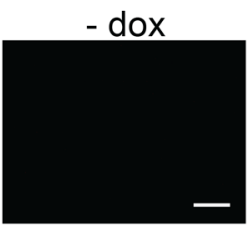

+ dox

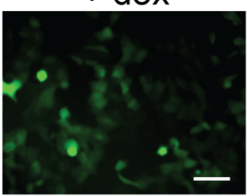

B

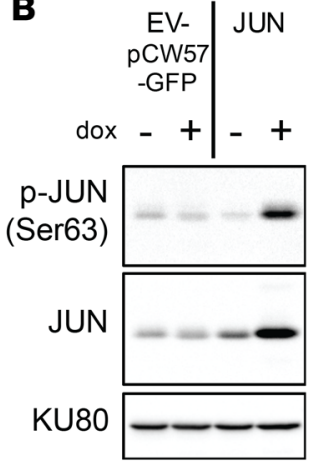

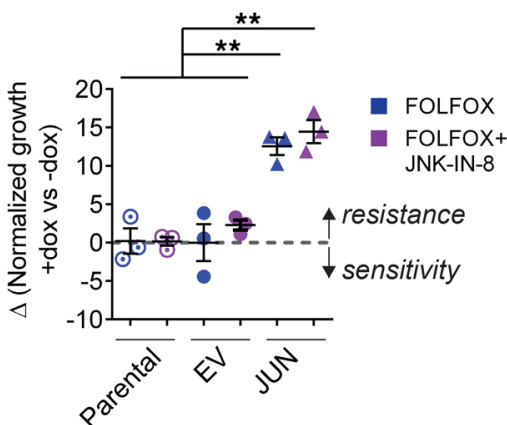

C
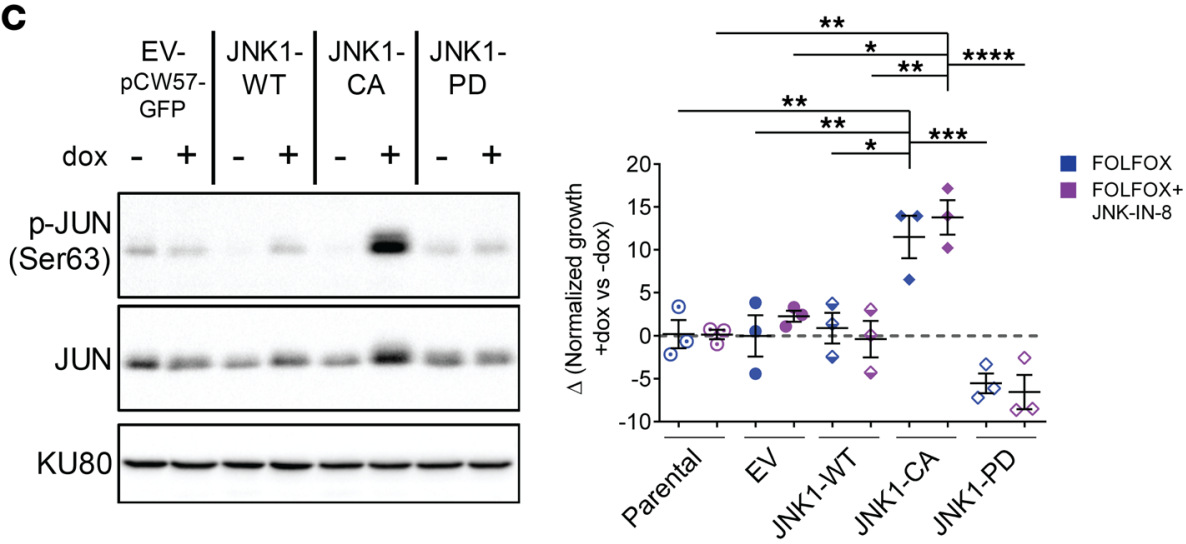

D
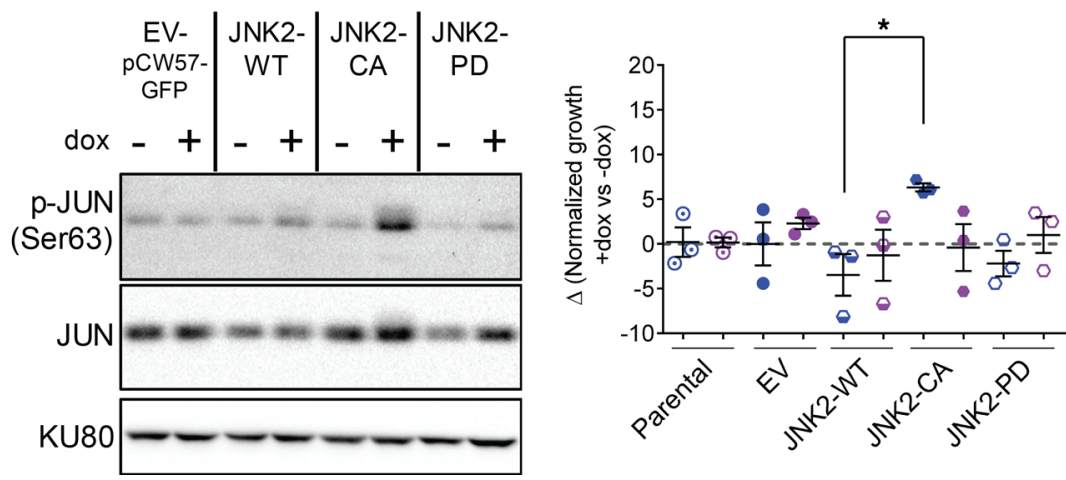

- FOLFOX

- FOLFOX+ JNK-IN-8

Figure 7. JUN and JNK1 are sufficient to induce FOLFOX resistance and abrogate synergy between FOLFOX and JNKIN-8. (A) Representative images of CFPAC-1 cells transduced with pCW57-GFP-P2A-neo after 24 hours without or with $1 \mu \mathrm{g} / \mathrm{mL}$ dox. Scale bars: $100 \mu \mathrm{m}$. (B) Representative immunoblot showing dox induction of JUN transgene or control cells infected with empty vector (EV). $\Delta$ (Normalized growth +dox versus -dox) values for each line after FOLFOX (blue) or FOLFOX + JNK-IN-8 (purple), as calculated by subtracting growth after FOLFOX ( $2 \mu \mathrm{M} 5$-FU + $0.2 \mu \mathrm{M} 0 \mathrm{OX}+10 \mu \mathrm{M} \mathrm{LEU})$ without dox from growth with FOLFOX with $1 \mu \mathrm{g} / \mu \mathrm{L}$ dox induction. Independent treatments and MTT assays were performed for FOLFOX versus FOLFOX + JNK-IN-8. Values shown are mean \pm SEM $(n=3)$. (C) JNK1 transgene and (D) JNK2 transgene overexpression in similar experiments to B. KU80 used as loading control.

To our knowledge, our study is the first to report in vivo effects of JNK-IN-8 in any patient-derived cancer model. We demonstrate the high specificity, rapid onset, and durability of JNK-IN-8 to inhibit JUN phosphorylation and enhance FOLFOX-induced tumor growth inhibition in a PDAC patient-derived organoid and tumor line at tolerable dosing and frequency of administration. Despite less marked synergy in vivo, across lines, the combination of FOLFOX and JNK-IN-8 led to tumor growth arrest and, in some cases, tumor regression near the end of the study period, indicating that longer treatment periods and additional dose combinations with alternative administration schedules may enhance in vivo synergy. Discrepancies between the observed synergy cell line compared with tumor xenografts highlight the known deficiencies in using a 2D cell culture as a proxy 
for in vivo responses in mouse models and human cancers $(46,47)$. Moreover, our studies in 2 patient-derived tumor lines cannot adequately capture the intertumoral heterogeneity characteristic of PDAC. The tumor linedependent variation in durability of response to JNK-IN-8 suggests that, as with other kinase inhibitors, adaptive signaling varies between lines following kinase inhibitor treatment. Comprehensive profiling of a larger number of patient-derived tumors and cell lines is crucial to detect associations between treatment response, pharmacodynamics, and baseline tumor kinome and transcriptome profiles such as PDAC tumor subtypes (54). Moreover, our studies did not assess the effects of the tumor microenvironment of immune and stromal cells that promote tumor progression via JNK signaling and may play important roles in determining in vivo treatment responses to the combination of chemotherapies with targeted kinase inhibitors (27).

Unlike in other cancers, specific roles for JNK1 and JNK2 had not been elucidated in PDAC, so we interrogated specific functions of these genes with lentiviral-mediated dox-inducible expression of JNK and JUN transgenes with different capacities for activity. Constitutively active JNK1 and JUN, but not JNK2, were able to heighten resistance to FOLFOX and abrogate synergy with JNK-IN-8, consistent with patient survival data that show a much stronger association between patient survival and JNK1 expression compared with JNK2. Selective inhibitors for JNK1 $(55,56)$ and JNK2 $(57)$ are emerging that will further clarify JNK1- and JNK2-specific effects and their potential as therapeutic targets.

Our results propose links between FOLFOX-mediated DNA damage, cell cycle responses, and synergy with JNK-IN-8. Low doses of FOLFOX led to profound S-phase arrest in lines displaying synergy and correspondingly robust activation of markers for DNA damage in CFPAC-1. Conversely, JUN activation, the cell cycle profile, and expression of DNA damage markers were less affected by FOLFOX in MIA PaCa-2 in association with limited synergy with JNK-IN-8. JUN is known to modulate cell cycle progression by transcriptionally repressing p53 and p21 tumor suppressor gene expression (22), but consistent with prior research in SP600125 (58), cell cycle effects of JNK-IN-8 were largely p21 independent, as evidenced by a lack of G1 arrest characteristic of p21-induced CDK2 inhibition after JNKIN-8. These findings suggest that JNK is necessary for preserving cell cycle arrest in the context of DNA damage caused by 5-FU and FOLFOX, and that JNK inhibition allows these damaged cells to progress through mitosis and ultimately undergo cell death.

This study establishes JUN activity downstream of JNK1 as a strong promoter of chemoresistance, but other JNK targets may also be important in the interaction between FOLFOX and JNK-IN-8, as indicated by residual resistance to FOLFOX following JUN knockdown and incomplete abrogation of synergy between FOLFOX and JNK-IN-8 following overexpression of JNK1 and JUN. JNK is known to cooperate with p38 stress-response signaling and mediate reactive oxygen species generation after cell stress (29, 33, 39), and JNK activates STAT3 and ATF2, along with other pathways involved in PDAC tumorigenesis and treatment responses (59-61). Further study is needed to isolate the pathways that interact to prime FOLFOX-treated cells for death when JNK and its downstream effectors are inhibited by JNK-IN-8.

In addition to encouraging further study of $\mathrm{JNK}$, our results also point to roles in chemoresistance for specific upstream kinases, many of which are sparsely studied in PDAC. For example, we found that MAP4K4, a canonical activator of the JNK signaling cascade $(19,36)$, was upregulated after 5-FU and FOLFOX by MIB-MS. MAP4K4 has been linked to PDAC cell invasion and proliferation (62), is overexpressed in PDAC tumors, and has been found to be associated with worse patient prognosis (63). Interestingly, the most upregulated family of kinases after 5-FU and FOLFOX were the TAOK family (TAOK1/2/3 = MAP3K16/17/18), kinases known to regulate p38-mediated stress response that also have poorly defined roles in JNK activation (19, 36-39). Recently discovered, specific inhibitors for MAP4K4 (64) and TAOK1/2 (65) merit further study for their JNK-dependent and -independent functions in PDAC chemoresistance.

Collectively, this study presents an unbiased methodology for identifying kinase signaling pathways involved in response and resistance to chemotherapies and identifies JNK1 and JUN as druggable drivers of chemoresistance to 5-FU and FOLFOX in PDAC. In the near future, such identification of tumor-specific signaling networks contributing to cancer progression and treatment responses has the potential to allow for patient-tailored treatments that can ultimately improve outcomes for this deadly disease.

\section{Methods}

Drugs and reagents. For in vivo studies, 5-FU, OX, and GEM were diluted in sterile $0.9 \%$ saline, and LEU was dissolved in sterile water. JNK-IN-8 (SelleckChem) was dissolved in sterile DMSO for in vitro studies and was then further dissolved in 15\% Tween 80 in sterile water for in vivo studies. 
PDX tumor expansions and treatments. PDAC tumors from deidentified patients with resected PDAC were stained with $\mathrm{H} \& \mathrm{E}$ to confirm histology, engrafted s.c. into the flanks of 6- to 8-week-old female NSG mice, and passaged as described previously (66). Pretreatment biopsies were taken when tumors reached $1 \mathrm{~cm}$ in any dimension. Treatment was initiated when biopsied tumors reached a median volume of 117 $\mathrm{mm}^{3}\left(68.75-239.06 \mathrm{~mm}^{3}\right)$. Treatments were assigned by rotation through all 4 experimental arms as tumors became available. FOLFOX (100 mg/mL LEU [Sagent], $50 \mathrm{mg} / \mathrm{mL} 5$-FU [Accord], and $5 \mathrm{mg} / \mathrm{mL}$ OX [Sagent]) and $0.9 \%$ saline vehicle treatments were administered IP $1 \times /$ week. GEM (Mylan) $(80 \mathrm{mg} / \mathrm{kg})$, JNK-IN-8 (30 mg/kg), and 15\% Tween $80+5 \%$ DMSO (both from Fisher) vehicle treatments were administered IP $2 \times$ /week. FOLFOX and GEM dosing was based on prior pharmacokinetics analyses (48). Animal body weights and tumor volumes $(\mathrm{V})$ were both measured $1 \times /$ week, with $\mathrm{V}=\left(\right.$ length $\times$ width $\left.{ }^{2}\right) / 2$. Treatment was continued for 28 days or when tumors reached $2 \mathrm{~cm}$ in any dimension. Harvested tumors were cut; half of each tumor was snap frozen in liquid nitrogen and stored at $-80^{\circ} \mathrm{C}$ until protein and/ or RNA isolation, while the other half was fixed in 10\% formalin and paraffin embedded (FFPE). FFPE tissue blocks were sectioned and stained with $\mathrm{H} \& \mathrm{E}$ for histopathological evaluation.

Cell and organoid culture. PDX-CLs and organoids were established from freshly harvested PDX tumor tissue, which was washed with DMEM/F12 media containing $1 \times$ antibiotic-antimycotic solution (Thermo Fisher Scientific), mechanically dissociated using a gentleMACS dissociator (Miltenyi Biotec), and incubated at $37^{\circ} \mathrm{C}$ in DMEM/F12 with collagenase + dispase (MilliporeSigma). Tumor suspensions were divided for organoid culture and PDX-CL establishment.

For organoid culture, dissociated tumor was resuspended in $90 \%$ Matrigel basement membrane matrix (Corning) and plated in approximately $120-\mu \mathrm{L}$ domes in twelve 96 -well plates. Organoid media consisted of (a1) 40\% DMEM/F12 growth media (Gibco), (b) 10\% FBS (Gibco), and (c) 50\% L-WRN conditioned DMEM/F12 media (+HEPES [Sigma], +GlutaMAX [Gibco], conditioned with Wnt-3A [ATCC CRL2647], R-spondin [Peprotech], and mNoggin [Peprotech]) containing growth factors: $0.2 \mu \mathrm{g} / \mathrm{mL} \mathrm{A83-}$ 01 (Tocris), $1 \times$ B-27 supplement (Invitrogen), $50 \mathrm{ng} / \mathrm{mL} \mathrm{mEGF}$ (Thermo Fisher Scientific), $100 \mathrm{ng} / \mathrm{mL}$ hFGF-10 (Gemini), $0.2 \mu \mathrm{g} / \mathrm{mL}$ Gastrin I (Tocris), $0.2 \mathrm{mg} / \mathrm{mL}$ N-acetylcysteine (MilliporeSigma), 1.22 $\mathrm{mg} / \mathrm{mL}$ nicotinamide (MilliporeSigma), and $3.4 \mu \mathrm{g} / \mathrm{mL}$ Y-27632 Rho kinase inhibitor (MilliporeSigma). Organoid media was replaced every 2-4 days for passaging or drug treatments (46).

For PDX-CLs, dissociated tumor was resuspended in warm 45\% DMEM/F12 media, 5\% FBS, and $50 \%$ L-WRN conditioned organoid media as described above but excluding Y-27632 Rho kinase inhibitor. Media was replaced daily for 1 week with decreasing concentrations of L-WRN conditioned organoid media and passaged when cells reached $70 \%-80 \%$ confluence. PDX-CL were confirmed as epithelial by positive IHC for cytokeratin 18 .

PDAC cell lines CFPAC-1 and MIA PaCa-2 were obtained from the American Type Culture Collection (ATCC) and authenticated via short-tandem repeat (STR) profiling (Genetica). All cell lines tested negative for mycoplasma monthly using MycoAlert (Lonza). Cell lines were cultured in DMEM/F12 (for patient-derived) or DMEM (for ATCC) medium with 10\% FBS, $100 \mathrm{U} / \mathrm{mL}$ penicillin (Sigma), and $100 \mu \mathrm{g} /$ $\mathrm{mL}$ streptomycin (all, unless noted, from Invitrogen) at $37^{\circ} \mathrm{C}$ in humidified $5 \% \mathrm{CO}_{2}$ atmosphere.

MIB chromatography and MS. MIB preparation and liquid chromatography-mass spectrometry (LC/MS) was carried out as previously described (34). Briefly, cell lysates were passed through columns containing broad-spectrum Type I kinase inhibitors (CTx-0294885, VI-16832, PP58 [all from Changchun Discovery Sciences LTD], Purvalanol B [Tocris], UNC-2147A, and UNC-8088A [Changchun Discovery Sciences LTD]) covalently attached to ECH- or EAH-activated Sepharose beads (GE Healthcare Life Sciences) to purify functional kinases. For assessing adaptive changes after chemotherapy treatments, peptides were labeled with iTRAQ; then, MS data were acquired with a MALDI TOF/TOF 5800 (AB Sciex) and analyzed by ProteinPilot Software Version 3.0 (AB Sciex) using the UniProtKB/Swiss-Prot data base. Proteins were accepted when $\geq 2$ unique peptides were identified at $99 \%$ confidence. MIB-MS profiles were performed for 2 biological replicate tumors or cell lines in each treatment arm. Changes in kinase binding between pretreatment and treated tumors, and cell lines were plotted in R 3.5.1 as mean \pm SEM. For MIB-MS competition studies to assess the JNK-IN-8 binding profile, peptide samples were analyzed by LC/MS/MS using an Easy nLC 1000 coupled to a QExactive mass spectrometer (Thermo Fisher Scientific). Data were analyzed using label-free quantification in MaxQuant version 1.5.1.2 with the integrated Andromeda search engine. FDR was set at 5\% and excluded kinases with only 1 unique peptide. 
RNA-seq. Total RNA (200-1000 ng) from pancreatic PDX tumors or cell lines was extracted using AllPrep DNA/RNA mini kit (Qiagen), and cDNA libraries were prepared with the TruSeq Stranded mRNA Sample Prep kit (Illumina). Sequencing of 75 bp paired end reads was completed on a NextSeq 500 high-output v2 flow cell kit (Illumina). BCL files were converted to FASTQ using bcl2fastq2 Conversion Software 2.20.0 and compiled into 1 FASTQ for each sample. Total read counts per gene were quantified using Salmon 0.9.1 using arguments "--gcBias --seqBias" (67).

For gene-level differential expression analysis, differential synergistic effects between FOLFOX and JNK-IN-8 were analyzed between CFPAC-1 and MIA PaCa-2 by constructing a negative binomial regression model for each gene to specify mean on a log scale and by assessing differential expression across treatments using DESeq2 1.20 .0 on R 3.5.1 $(49,68)$.

Immunoblotting. Cells in culture were washed with PBS, harvested by scraping, and prepared as whole cell lysates or fractionated into cytosolic and chromatin-bound fractions. Whole cell lysates were prepared using Pierce RIPA buffer (Thermo Fisher Scientific) containing phosphatase inhibitor cocktail sets I/II (Calbiochem) and complete protease inhibitor (MilliporeSigma). Fractionation was performed with CSK buffer (pH 7, $10 \mathrm{mM}$ PIPES sodium salt [Fisher], $100 \mathrm{mM} \mathrm{NaCl}$ [Fisher], $300 \mathrm{mM}$ sucrose [Sigma], $3 \mathrm{mM}$ $\mathrm{MgCl}_{2}$ [VWR], $1 \mathrm{mM}$ EGTA [Sigma], 0.1\% Triton X-100 [Fisher]) containing phosphatase inhibitor cocktail sets I and II and complete protease inhibitor. Cytosolic fractions were collected after mixing whole cell lysates with CSK buffer and centrifuging at $1500 \mathrm{~g}$ for 4 minutes at $4^{\circ} \mathrm{C}$. The remaining chromatin-bound pellet was cleaned by resuspension in CSK buffer, followed by centrifugation at $1500 \mathrm{~g}$ for 4 minutes at $4^{\circ} \mathrm{C}$ and resuspension in CSK buffer with $0.1 \%$ benzonase (Thermo Fisher Scientific).

Lysate protein concentrations were measured with $2 \mathrm{mg} / \mathrm{mL}$ albumin standard (Thermo Fisher Scientific) and Bradford reagent (MilliporeSigma). Whole cell lysates (20-40 $\mu \mathrm{g}$ ) or chromatin-bound fractions $(5-40 \mu \mathrm{g})$ were electrophoresed on $8 \%-10 \%$ SDS polyacrylamide (Fisher) gels and electrotransferred to PVDF membranes (Thermo). Transferred membranes were blocked with 5\% nonfat dried milk (Fisher) in $1 \times$ Tris-buffered saline (Fisher) with $0.1 \%$ Tween 20 (Fisher) $(1 \times$ TBST) and incubated overnight at $4^{\circ} \mathrm{C}$ with 1:500 dilutions of p-JNK (Thr183/Tyr185) antibody, or 1:1,000 dilutions of other primary antibodies in 5\% BSA (Fisher) in $1 \times$ TBST. Following washing, membranes were incubated with a 1:10,000 dilution of HRP-conjugated goat anti-rabbit (catalog 31462) or antimouse (catalog 31432) secondary antibody (Invitrogen) in 5\% nonfat dried milk in $1 \times$ TBST for 1 hour. Immunoreactive bands were detected using Amersham ECL Prime detection agent (GE Healthcare). Intensity of immunoreactive bands were quantified by densitometry using Imagelab 6.0.1 (BioRad) and normalized to vehicle-treated controls. Stripping of bands between detection of proteins of similar sizes was accomplished by 15-minute incubation with Restore Stripping Buffer (Thermo Fisher Scientific) followed by washing and blocking as above. Antibodies against p-JNK1/2 (Thr183/Tyr185, catalog 4668S), JNK1 (mouse, catalog 3708S), JNK2 (catalog 9258S), p-JUN (Ser63, catalog 2361S), JUN (catalog 9165S), p-CHK1 (Ser317, catalog 2344), p-CHK2 (Thr68, catalog 2197), and CHK2 (catalog 6334) were from Cell Signaling Technology, while p-ATM (mouse, catalog 47739), ATM (catalog 377293), PCNA (mouse, catalog 56), FANCD2 (catalog 20022), cyclin E (catalog 247), CHK1 (catalog 8408), and ACTB HRP (catalog 4778) antibodies were from Santa Cruz Biotechnology Inc. KU80 (catalog GTX70485) was from GeneTex. p-ATM, PCNA, FANCD2, p-H3, and p-H2AX were assessed in chromatin-bound fractions.

Transwell invasion assays. Twenty-four-well plate inserts with $8-\mu \mathrm{m}$ pores (Greiner) were coated with $100 \mu \mathrm{L}$ Matrigel, incubated at $37^{\circ} \mathrm{C}$ for 2 hours, and placed into wells containing $750 \mu \mathrm{L}$ normal culture media. A total of $5 \times 10^{4}$ (CFPAC-1) or $2 \times 10^{5}$ (MIA PaCa-2) cells were added to each coated insert by suspending cells in $200 \mu \mathrm{L}$ DMEM with $1 \%$ FBS and the indicated dose of JNK-IN-8. Cells were allowed to invade through the matrix and underlying pores for 16 hours; they then were fixed and stained using DiffQuik kit (Thermo Fisher Scientific). Invasion was quantified as the percent of the insert covered by invading cells, as determined by imaging stained cells and filtering by color of stained nuclei with a BZ-X710 fluorescent microscope (Keyence).

Synergy screen. Growth inhibition following combinations of FOLFOX with and without a library of kinase and other small molecule inhibitors was performed by plating PDX-CLs in 384-well plates in 40 $\mu \mathrm{L}$ at 2000 cells/well, allowing cells to adhere overnight, and then treating with 176 individual drugs at 5 doses alone and in combination with 3 doses of FOLFOX $(2 \mu \mathrm{M}$ 5-FU $+0.2 \mu \mathrm{M}$ OX; $20 \mu \mathrm{M}$ 5-FU +2 $\mu \mathrm{M}$ OX; and $200 \mu \mathrm{M} 5$-FU $+20 \mu \mathrm{M} \mathrm{OX}$ ). After 72 hours, cell viability was quantified using CellTiter-Glo 
Luminescent Cell Viability Assay (Promega). Growth was normalized to wells treated with DMSO vehicle (100\% viability) and Bortezomib (0\% viability). Synergy was scored using Bliss independence modelling as described below.

MTT growth assay. For drug studies and dox-inducible expression experiments, $1 \times 10^{3}$ to $5 \times 10^{3}$ cells were plated in $90 \mu \mathrm{L}$ culture medium and cultured overnight in quadruplicate. For drug studies, media containing PBS vehicle (for FOLFOX), DMSO vehicle (for JNK-IN-8), FOLFOX (0-40 $\mu \mathrm{M}$ ), and JNK-IN-8 $(0-40 \mu \mathrm{M})$ were added to the indicated final concentrations. After 72 -hour incubation, $50 \mu \mathrm{L}$ of $5 \mathrm{mg} / \mathrm{mL}$ MTT (MilliporeSigma) dissolved in PBS at pH 7.4 was added to each well. After 1 hour, culture medium and MTT reagent was discarded, and $200 \mu \mathrm{L}$ dimethyl sulfoxide was added to each well and mixed thoroughly. Absorbance at OD $560 \mathrm{~nm}$ was measured using a Synergy 2 plate reader (BioTek). Cell viability at each drug concentration was calculated according to the formula: $100 \% \times($ experimental OD 560/vehicle OD 560). All assays were performed in triplicate.

For dox-inducible expression experiments, both dox-induced and no-dox control cells were seeded from a single population of plasmid-transduced cell lines. After cells adhered, half were treated with dox; then, after 8 hours, all cells were treated with FOLFOX and/or JNK-IN-8. Viability was assessed after 72 hours by MTT assay as described above.

Crystal violet growth assay. A total of $1 \times 10^{3}$ to $5 \times 10^{3}$ cells were plated in triplicate at very low density in 6-well plates overnight; they were then treated with indicated drugs every 3 days for 15 days, total. Colonies were visualized by methanol fixation, followed by 20 -minute rocking incubation with filtered $20 \%$ methanol in distilled water containing $0.5 \%$ crystal violet powder (MilliporeSigma) and repeated gentle washing with water, air drying, and imaging (Canon LiDE 90).

Organoid CellTiter-Glo growth assay. Organoids were grown in Matrigel domes in 24-well plates until they reached about $70 \%$ confluence. Organoids were collected by resuspension of domes with normal culture media, fragmentation with a 25-gauge needle, and centrifugation at 2,000 $\mathrm{g}$ for 5 minutes at room temperature. Media was aspirated, the resulting organoid pellet was diluted by a factor of 4 in fresh Matrigel containing $20 \%$ organoid media, and $50 \mu \mathrm{L}$ of resuspended organoids was plated in 96-well plates in triplicate for each dose. After 30 minutes at $37^{\circ} \mathrm{C}, 70 \mu \mathrm{L}$ of fresh organoid media was added, and organoids were cultured overnight, after which indicated doses were added to a final volume of $100 \mu \mathrm{L} /$ well. After 5 days of growth, images were captured with a BZ-X710 fluorescent microscope (Keyence), and organoid viability was assessed with CellTiter-Glo 3D (Promega) and Synergy 2 plate reader (BioTek).

Flow cytometry. After 72 hours of the indicated treatments, cells in culture were pulsed with $10 \mu \mathrm{M}$ BrdU (MilliporeSigma) for 1 hour at $37^{\circ} \mathrm{C}$, trypsinized, and fixed in $65 \% \mathrm{DMEM} / 35 \%$ ethanol solution for 1-4 days. DNA was denatured by 20-minute incubation at room temperature in $1 \mathrm{~mL} 2 \mathrm{M} \mathrm{HCl}$. Cells were washed in $0.1 \mathrm{M}$ sodium borate, $\mathrm{pH} 8.5$, followed by PBS and was then incubated in the dark for 30 minutes in FITC-conjugated anti-BrdU (BD Pharmingen, 347583) in PBS with 0.3\% Tween-20 and 0.3\% BSA. Cells were resuspended in PBS containing $8 \mu \mathrm{g} / \mathrm{mL}$ RNAse A (Qiagen) and $10 \mu \mathrm{g} / \mathrm{mL}$ PI (BioLegend) and incubated in the dark for 30 minutes. Flow analysis was conducted on an Accuri C6 flow cytometer with C6 Plus software (BD Biosciences).

Cloning inducible expression vectors. To generate cell lines with inducible, reversible expression of $J U N$ and $J N K$ constructs, cloning was performed using the In-Fusion HD Cloning Kit (Takara). For inducible overexpression, the backbone plasmid pCW57-GFP-P2A-MCS-neo (Addgene, 89181) was digested with BamHI-HF (New England Biolabs), and coding sequences (CDS) were PCR amplified from plasmids from Addgene (JNK1a1, 13798; JNK2a2, 13755; MKK7b2-JNK1a1, 19726; MKK7b2-JNK2a2, 19727; MKK7b2-JNK1a1-APF, 19730; MKK7b2-JNK2a2-APF, 19726 and 13761; JUN, 40348) using primers to generate CDS sequences with homologous regions with the cut backbone (JNK1a1 forward 5'-ACGCGTTGTACAGGATCCAGCAGAAGCAAGCGTGACAACA-3'; JNK1a1 reverse 5'-TTAGCACAGGTGCAGCAGTGAGGATCCGGGGTTGGGGTT-3'; JNK2a2 forward 5'-ACGCGTTGTACAGGATCCAGCGACAGTAAATGTGACAGTCAGT-3'; JNK2a2 reverse 5'-CCCTTGAAGGCTGTCGATGAGGATCCGGGGTTGGGGTT-3'; MKK7b2-JNK1a1 forward 5'-ACGCGTTGTACAGGATCCGCGGCGTCCTCCCTG-3'; MKK7b2-JNK1a1 reverse - same as JNK1a1 reverse; MKK7b2-JNK2a2 forward - same as MKK7b2-JNK1a1 forward; MKK7b2-JNK2a2 reverse - same as JNK2a2 reverse; MKK7b2-JNK1a1-APF forward - same as MKK7b2-JNK1a1 forward; MKK7b2-JNK1a1-APF reverse - same as JNK1a1 reverse; MKK7b2-JNK2a2-APF forward \#1 - same as MKK7b2-JNK1a1 forward; MKK7b2-JNK2a2-APF reverse \#1 5'-GAGTTACCGTAAAGGATC- 
CATGAGCGACAGT-3'; MKK7b2-JNK2a2-APF forward \#2 5'-GGATCCATGAGCGACAGTAAATGTGACAGT-3'; MKK7b2-JNK2a2-APF reverse \#2 - same as JNK2a2 reverse; JUN forward 5'-ACGCGTTGTACAGGATCCACTGCAAAGATGGAAACGACCTT-3'; JUN reverse 5'-ACGCAGCAGTTGCAAACATTTTGAGGATCCGGGGTTGGGGTT-3'). All cloned plasmids were verified by Sanger sequencing (Eton Biosciences).

Lentivirus production and transduction. Cloned plasmids were delivered to cells by generating replication-incompetent lentivirus in 293T cells with transfection of X-tremeGENE 9 (MilliporeSigma), psPAX2, and pCMV VSV-G, along with the corresponding reporter plasmid. For inducible overexpression experiments, reporter plasmids were recombinant pCW57-GFP plasmids, as described above. For shRNA experiments, a validated shRNA against the 3'UTR of the JUN mRNA (MilliporeSigma, TRCN0000355647) and EV control pLKO.1-puro (Addgene, 8453) were used as reporter plasmids. Following 24-hour incubation, media were replaced with DMEM supplanted with $30 \%$ FBS. Lentivirus was harvested at both 48 and 72 hours after transfection by collecting media, centrifugation at $2000 \mathrm{~g}$ for 5 minutes at room temperature to remove $293 \mathrm{~T}$ cells and debris, filtration through $0.45-\mu \mathrm{m}$ sterile PES membranes, and storage at $-80^{\circ} \mathrm{C}$. For transduction with lentivirus, $1.5 \times 10^{6} \mathrm{CFPAC}-1$ or MIA $\mathrm{PaCa}-2$ cells were seeded in $100-\mathrm{mm}$ plates and transduced with lentivirus containing the appropriate overexpression plasmid, along with $8 \mu \mathrm{g} / \mathrm{mL}$ polybrene (MilliporeSigma). After 24 hours, medium was replaced and cells were cultured for another 4 days with $2 \mu \mathrm{g} / \mathrm{mL}$ puromycin (Corning) or 14 days with $800 \mu \mathrm{g} / \mathrm{mL}$ geneticin (Thermo Fisher Scientific) before cryopreservation and experimentation.

Statistics. Library compound screen $\sum \Delta$ Bliss values were calculated assuming Bliss independence between drugs, where responses to combinations of 2 drugs equal the sum of the 2 fractional responses of individual drugs minus their product, where $(\mathrm{Fa}+\mathrm{Fb})-(\mathrm{Fa} \times \mathrm{Fb})$. $\Delta$ Bliss is zero for a given dose combination when there is no overall synergy or antagonism, while negative $\Delta$ Bliss values indicate synergy at that combination. Overall synergy score for a drug combination was calculated by $\sum \Delta$ Bliss.

Patient survival analysis was performed using the previously published cohort of 146 patients in TCGA pancreatic adenocarcinoma (PAAD) data set, excluding patients with evidence of metastatic disease (M1) at diagnosis (42). Patients expressing distinctly high levels (top 10\%) of JNK1 or JNK2 were classified as the high-expression group, with survival compared with remaining patients using the "survminer" package in R 3.5.1. Survival was plotted using the Kaplan-Meier product-limit method, and significance of tumor gene expression differences was measured by Mantel-Cox log-rank test, with statistical significance defined as $P$ $<0.01$. Hazard ratio (HR) was determined by Cox proportional-hazards model. To assess the association of JUN transcription factor activity with patient outcome, a JUN signature for each sample in the TCGA PAAD data set was calculated as mean of expression rankings of predicted JUN target genes (MSigDB CREBP1CJUN_01) (40). Samples were then divided by median JUN signature scores and subjected to survival analysis, as described above.

Statistical significance in MTT assays for synergy and overexpression-mediated changes was determined across replicate experiments using 1-way ANOVA analysis with Tukey's multiple comparisons test. For shRNA experiments, statistical significance was determined using 2-way ANOVA with Fisher's multiple comparison's test. Data were normalized and plotted in Prism as mean \pm SEM (Graphpad), and synergy was assessed using CompuSyn (45). For transwell invasion assays, significance was determined using 1-way ANOVA with Holm-Sidak's multiple comparisons test.

For in vivo tumor growth inhibition studies, sample sizes were estimated to detect a $30 \%$ difference in volumes between treatment arms with $95 \%$ CI based on variances in historical growth of each tumor line. Volume changes were analyzed for significance using 1-way ANOVA with Dunnett's multiple comparisons test in Prism (Graphpad).

Determination of differentially expressed genes following RNA-seq was performed using DESeq2 1.20.0 on $\mathrm{R}$ 3.5.1 with a mean structure involving a linear combination of intercepts, main effects, and second-order interactions between cell lines, FOLFOX, and JNK-IN-8 $(49,68)$. Third-order interactions were not assessed due to insufficient power. To model the 2 doses of FOLFOX while retaining maximum degrees of freedom, the low dose was set to have $80 \%$ of the effect on gene expression changes as the high dose. Other coefficients besides $80 \%$ did not meaningfully affect results by a sensitivity analysis. Next, pathway-level significance was assessed using goseq 1.32.0, which performs Gene Ontology (GO) analysis while accounting for selection bias introduced by enrichment of longer genes among significantly differentially expressed genes (69). Plots were made with normalized expression of genes changed by the 
second-order FOLFOX $\times$ JNK-IN-8 effect (with FDR $<0.2$ without independent filtering in DESeq2). Heatmaps and consensus clustering, when shown, were performed via row-normalized gene expression with heatmap.3 (https://www.rdocumentation.org/packages/GMD/versions/0.3.3/topics/heatmap.3).

Statistical significance is represented throughout as ${ }^{*} P<0.05$, ${ }^{* *} P<0.01,{ }^{* *} P<0.001$, and ${ }^{* * * *} P<0.0001$.

Study approval. PDAC tumors from deidentified patients with resected PDAC were obtained from the UNC IRB-approved Tissue Procurement Facility after IRB approval (no. 08-1153). All animal experiments were carried out in accordance with NIH protocols and approved by the UNC IACUC (ID 15-319.0-C).

\section{Author contributions}

MBL and JJY conceived of the research hypotheses and wrote the manuscript. MBL, SGHL, and BTG performed and analyzed the compound library synergy screen. MBL, XLP, MPE, NUR, RAM, LMG, and JJY performed and analyzed MIB-MS experiments. MBL, XLP, CJ, and ABM performed and analyzed RNA-seq. MBL and YX performed immunoblots. MBL and ABM performed transwell invasion assays. MBL and YG performed flow cytometry experiments. MBL completed all other experiments. LMG, GLJ, and CV contributed reagents, equipment, and lab space for MIB-MS, compound library screen, and flow cytometry experiments. All authors critically discussed the results and developing manuscript throughout the project.

\section{Acknowledgments}

The authors thank H. Shelton Earp, Shawn Gomez, and Ben Major for their expertise and guidance. We also thank Charlene M. Santos and the Lineberger Comprehensive Cancer Center Animal Studies Core, the UNC PDX Program, the UNC Tissue Procurement Facility, and the UNC Translational Pathology Laboratory for all their expertise and technical assistance. This work was supported by NIH R01-CA193650 and R01-CA199064. MBL was supported by NIH 5-F30-CA213916 and the UNC Department of Pharmacology NIH T32 training grant.

Address correspondence to: Jen Jen Yeh, UNC Lineberger Comprehensive Cancer Center, 21-205 LCCC, CB\# 7295, 450 West Drive, Chapel Hill, North Carolina 27599-7295, USA. Phone: 919.966.5221; Email: jjyeh@med.unc.edu.

1. Cronin KA, et al. Annual Report to the Nation on the Status of Cancer, part I: National cancer statistics. Cancer. 2018;124(13):2785-2800.

2. Siegel RL, Miller KD, Jemal A. Cancer statistics, 2018. CA Cancer J Clin. 2018;68(1):7-30.

3. Gebbia V, et al. Second-line chemotherapy in advanced pancreatic carcinoma: a multicenter survey of the Gruppo Oncologico Italia Meridionale on the activity and safety of the FOLFOX4 regimen in clinical practice. Ann Oncol. 2007;18 Suppl 6:vi124-vi127.

4. Oettle $\mathrm{H}$, et al. Second-line oxaliplatin, folinic acid, and fluorouracil versus folinic acid and fluorouracil alone for gemcitabine-refractory pancreatic cancer: outcomes from the CONKO-003 trial. J Clin Oncol. 2014;32(23):2423-2429.

5. Schwarz L, et al. Resectable pancreatic adenocarcinoma neo-adjuvant FOLF(IRIN)OX-based chemotherapy - a multicenter, non-comparative, randomized, phase II trial (PANACHE01-PRODIGE48 study). BMC Cancer. 2018;18(1):762.

6. Gourgou-Bourgade S, et al. Impact of FOLFIRINOX compared with gemcitabine on quality of life in patients with metastatic pancreatic cancer: results from the PRODIGE 4/ACCORD 11 randomized trial. J Clin Oncol. 2013;31(1):23-29.

7. Abendroth A, et al. Long-term outcome of patients with advanced pancreatic cancer treated with sequential chemotherapies before the era of modern combination therapy protocols. J Cancer Res Clin Oncol. 2019;145(2):445-455.

8. Tempero MA, et al. Pancreatic Adenocarcinoma, Version 2.2017, NCCN Clinical Practice Guidelines in Oncology. J Natl Compr Canc Netw. 2017;15(8):1028-1061.

9. Suker M, et al. FOLFIRINOX for locally advanced pancreatic cancer: a systematic review and patient-level meta-analysis. Lancet Oncol. 2016;17(6):801-810.

10. Conroy T, et al. FOLFIRINOX versus gemcitabine for metastatic pancreatic cancer. N Engl J Med. 2011;364(19):1817-1825.

11. Muranaka T, et al. Comparison of efficacy and toxicity of FOLFIRINOX and gemcitabine with nab-paclitaxel in unresectable pancreatic cancer. J Gastrointest Oncol. 2017;8(3):566-571.

12. Ho MY, Kennecke HF, Renouf DJ, Cheung WY, Lim HJ, Gill S. Defining Eligibility of FOLFIRINOX for First-Line Metastatic Pancreatic Adenocarcinoma (MPC) in the Province of British Columbia: A Population-based Retrospective Study. Am J Clin Oncol. 2017;40(6):552-554.

13. Wang WB, Yang Y, Zhao YP, Zhang TP, Liao Q, Shu H. Recent studies of 5-fluorouracil resistance in pancreatic cancer. World J Gastroenterol. 2014;20(42):15682-15690.

14. Amrutkar M, Gladhaug IP. Pancreatic Cancer Chemoresistance to Gemcitabine. Cancers (Basel). 2017;9(11):E157.

15. Krulikas LJ, et al. Application of Integrated Drug Screening/Kinome Analysis to Identify Inhibitors of Gemcitabine-Resistant Pancreatic Cancer Cell Growth. SLAS Discov. 2018;23(8):850-861.

16. Zhang T, et al. Discovery of potent and selective covalent inhibitors of JNK. Chem Biol. 2012;19(1):140-154. 
17. Dérijard B, et al. JNK1: a protein kinase stimulated by UV light and Ha-Ras that binds and phosphorylates the c-Jun activation domain. Cell. 1994;76(6):1025-1037.

18. Chen YR, Wang X, Templeton D, Davis RJ, Tan TH. The role of c-Jun N-terminal kinase (JNK) in apoptosis induced by ultraviolet $\mathrm{C}$ and gamma radiation. Duration of JNK activation may determine cell death and proliferation. J Biol Chem. 1996;271(50):31929-31936.

19. Davis RJ. Signal transduction by the JNK group of MAP kinases. Cell. 2000;103(2):239-252.

20. Johnson R, Spiegelman B, Hanahan D, Wisdom R. Cellular transformation and malignancy induced by ras require c-jun. $M o l$ Cell Biol. 1996;16(8):4504-4511.

21. Gupta S, et al. Selective interaction of JNK protein kinase isoforms with transcription factors. EMBO J. 1996;15(11):2760-2770.

22. Shaulian E, Karin M. AP-1 in cell proliferation and survival. Oncogene. 2001;20(19):2390-2400.

23. Davies CC, et al. Impaired JNK signaling cooperates with KrasG12D expression to accelerate pancreatic ductal adenocarcinoma. Cancer Res. 2014;74(12):3344-3356.

24. Xu Z, et al. Sophoridine induces apoptosis and S phase arrest via ROS-dependent JNK and ERK activation in human pancreatic cancer cells. J Exp Clin Cancer Res. 2017;36(1):124.

25. Okada M, et al. Targeting the K-Ras--JNK axis eliminates cancer stem-like cells and prevents pancreatic tumor formation. Oncotarget. 2014;5(13):5100-5112.

26. Takahashi R, et al. Therapeutic effect of c-Jun N-terminal kinase inhibition on pancreatic cancer. Cancer Sci. 2013;104(3):337-344.

27. Sato T, et al. c-Jun N-terminal kinase in pancreatic tumor stroma augments tumor development in mice. Cancer Sci. 2017;108(11):2156-2165.

28. Tessari G, et al. The expression of proto-oncogene c-jun in human pancreatic cancer. Anticancer Res. 1999;19(1B):863-867.

29. Suzuki S, et al. JNK suppression of chemotherapeutic agents-induced ROS confers chemoresistance on pancreatic cancer stem cells. Oncotarget. 2015;6(1):458-470.

30. Recio-Boiles A, et al. JNK pathway inhibition selectively primes pancreatic cancer stem cells to TRAIL-induced apoptosis without affecting the physiology of normal tissue resident stem cells. Oncotarget. 2016;7(9):9890-9906.

31. Bain J, McLauchlan H, Elliott M, Cohen P. The specificities of protein kinase inhibitors: an update. Biochem J. 2003;371(Pt 1):199-204

32. Kim JA, Lee J, Margolis RL, Fotedar R. SP600125 suppresses Cdk1 and induces endoreplication directly from G2 phase, independent of JNK inhibition. Oncogene. 2010;29(11):1702-1716.

33. Staples CJ, Owens DM, Maier JV, Cato AC, Keyse SM. Cross-talk between the p38alpha and JNK MAPK pathways mediated by MAP kinase phosphatase-1 determines cellular sensitivity to UV radiation. J Biol Chem. 2010;285(34):25928-25940.

34. Duncan JS, et al. Dynamic reprogramming of the kinome in response to targeted MEK inhibition in triple-negative breast cancer. Cell. 2012;149(2):307-321

35. Stuhlmiller TJ, et al. Inhibition of Lapatinib-Induced Kinome Reprogramming in ERBB2-Positive Breast Cancer by Targeting BET Family Bromodomains. Cell Rep. 2015;11(3):390-404.

36. Su YC, Han J, Xu S, Cobb M, Skolnik EY. NIK is a new Ste20-related kinase that binds NCK and MEKK1 and activates the SAPK/JNK cascade via a conserved regulatory domain. EMBO J. 1997;16(6):1279-1290.

37. Zihni C, Mitsopoulos C, Tavares IA, Ridley AJ, Morris JD. Prostate-derived sterile 20-like kinase 2 (PSK2) regulates apoptotic morphology via C-Jun N-terminal kinase and Rho kinase-1. J Biol Chem. 2006;281(11):7317-7323.

38. Huangfu WC, Omori E, Akira S, Matsumoto K, Ninomiya-Tsuji J. Osmotic stress activates the TAK1-JNK pathway while blocking TAK1-mediated NF-kappaB activation: TAO2 regulates TAK1 pathways. J Biol Chem. 2006;281(39):28802-28810.

39. Win S, Than TA, Kaplowitz N. The Regulation of JNK Signaling Pathways in Cell Death through the Interplay with Mitochondrial SAB and Upstream Post-Translational Effects. Int J Mol Sci. 2018;19(11):E3657.

40. Liberzon A, Subramanian A, Pinchback R, Thorvaldsdóttir H, Tamayo P, Mesirov JP. Molecular signatures data base (MSigDB) 3.0. Bioinformatics. 2011;27(12):1739-1740.

41. Subramanian A, et al. Gene set enrichment analysis: a knowledge-based approach for interpreting genome-wide expression profiles. Proc Natl Acad Sci USA. 2005;102(43):15545-15550.

42. Cancer Genome Atlas Research Network. Integrated Genomic Characterization of Pancreatic Ductal Adenocarcinoma. Cancer Cell. 2017;32(2):185-203.e13.

43. Baillie TA. Targeted Covalent Inhibitors for Drug Design. Angew Chem Int Ed Engl. 2016;55(43):13408-13421.

44. Ozanne BW, Spence HJ, McGarry LC, Hennigan RF. Transcription factors control invasion: AP-1 the first among equals. Oncogene. 2007;26(1):1-10.

45. Chou TC. Theoretical basis, experimental design, and computerized simulation of synergism and antagonism in drug combination studies. Pharmacol Rev. 2006;58(3):621-681.

46. Boj SF, et al. Organoid models of human and mouse ductal pancreatic cancer. Cell. 2015;160(1-2):324-338.

47. Gao H, et al. High-throughput screening using patient-derived tumor xenografts to predict clinical trial drug response. Nat Med. 2015;21(11):1318-1325.

48. Byrne JD, et al. Iontophoretic device delivery for the localized treatment of pancreatic ductal adenocarcinoma. Proc Natl Acad Sci USA. 2016;113(8):2200-2205.

49. Love MI, Huber W, Anders S. Moderated estimation of fold change and dispersion for RNA-seq data with DESeq2. Genome Biol. 2014;15(12):550.

50. Kirschner K, Melton DW. Multiple roles of the ERCC1-XPF endonuclease in DNA repair and resistance to anticancer drugs. Anticancer Res. 2010;30(9):3223-3232.

51. Chakrabarti G, et al. Tumor-selective use of DNA base excision repair inhibition in pancreatic cancer using the NQO1 bioactivatable drug, $\beta$-lapachone. Sci Rep. 2015;5:17066.

52. Picco V, Pagès G. Linking JNK Activity to the DNA Damage Response. Genes Cancer. 2013;4(9-10):360-368.

53. Van Meter M, et al. JNK Phosphorylates SIRT6 to Stimulate DNA Double-Strand Break Repair in Response to Oxidative Stress by Recruiting PARP1 to DNA Breaks. Cell Rep. 2016;16(10):2641-2650.

54. Moffitt RA, et al. Virtual microdissection identifies distinct tumor- and stroma-specific subtypes of pancreatic ductal adenocar- 
cinoma. Nat Genet. 2015;47(10):1168-1178.

55. Yao K, et al. A selective small-molecule inhibitor of c-Jun N-terminal kinase 1. FEBS Lett. 2009;583(13):2208-2212.

56. Qiu C, et al. Licochalcone A Inhibits the Proliferation of Human Lung Cancer Cell Lines A549 and H460 by Inducing G2/M Cell Cycle Arrest and ER Stress. Int J Mol Sci. 2017;18(8):E1761.

57. Kaoud TS, et al. Development of JNK2-selective peptide inhibitors that inhibit breast cancer cell migration. ACS Chem Biol. 2011;6(6):658-666.

58. Kuntzen C, et al. Inhibition of c-Jun-N-terminal-kinase sensitizes tumor cells to CD95-induced apoptosis and induces G2/M cell cycle arrest. Cancer Res. 2005;65(15):6780-6788.

59. Hayakawa J, Depatie C, Ohmichi M, Mercola D. The activation of c-Jun NH2-terminal kinase (JNK) by DNA-damaging agents serves to promote drug resistance via activating transcription factor 2 (ATF2)-dependent enhanced DNA repair. J Biol Chem. 2003;278(23):20582-20592.

60. Bogoyevitch MA, Kobe B. Uses for JNK: the many and varied substrates of the c-Jun N-terminal kinases. Microbiol Mol Biol Rev. 2006;70(4):1061-1095

61. Gkouveris I, Nikitakis N, Karanikou M, Rassidakis G, Sklavounou A. JNK1/2 expression and modulation of STAT3 signaling in oral cancer. Oncol Lett. 2016;12(1):699-706.

62. Zhao G, et al. miRNA-141, downregulated in pancreatic cancer, inhibits cell proliferation and invasion by directly targeting MAP4K4. Mol Cancer Ther. 2013;12(11):2569-2580.

63. Liang JJ, et al. Expression of MAP4K4 is associated with worse prognosis in patients with stage II pancreatic ductal adenocar cinoma. Clin Cancer Res. 2008;14(21):7043-7049.

64. Dow RL, et al. 2-Aminopyridine-Based Mitogen-Activated Protein Kinase Kinase Kinase Kinase 4 (MAP4K4) Inhibitors: Assessment of Mechanism-Based Safety. J Med Chem. 2018;61(7):3114-3125.

65. Koo CY, et al. Targeting TAO Kinases Using a New Inhibitor Compound Delays Mitosis and Induces Mitotic Cell Death in Centrosome Amplified Breast Cancer Cells. Mol Cancer Ther. 2017;16(11):2410-2421.

66. Lipner MB, et al. Metformin Treatment Does Not Inhibit Growth of Pancreatic Cancer Patient-Derived Xenografts. PLoS ONE. 2016;11(1):e0147113.

67. Patro R, Duggal G, Love MI, Irizarry RA, Kingsford C. Salmon provides fast and bias-aware quantification of transcript expression. Nat Methods. 2017;14(4):417-419.

68. R Core Team. The R Project for Statistical Computing. R-project. https://www.R-project.org/. Accessed March $26,2020$.

69. Young MD, Wakefield MJ, Smyth GK, Oshlack A. Gene ontology analysis for RNA-seq: accounting for selection bias. Genome Biol. 2010;11(2):R14. 